\title{
Turbulent shear-layer mixing at high Reynolds numbers: effects of inflow conditions
}

\author{
By M. D. SLESSOR, C. L. BOND AND P. E. DIMOTAKIS \\ Graduate Aeronautical Laboratories, California Institute of Technology, Pasadena, CA 91125, USA
}

(Received 9 February 1998 and in revised form 29 June 1998)

We report on the results from a set of incompressible, shear-layer flow experiments, at high Reynolds number $\left(\operatorname{Re}_{\delta} \equiv \rho \Delta U \delta_{T}(x) / \mu \simeq 2 \times 10^{5}\right)$, in which the inflow conditions of shear-layer formation were varied $\left(\delta_{T}\right.$ is the temperature-rise thickness for chemically-reacting shear layers). Both inert and chemically-reacting flows were investigated, the latter employing the $\left(\mathrm{H}_{2}+\mathrm{NO}\right) / \mathrm{F}_{2}$ chemical system in the kineticallyfast regime to measure molecular mixing. Inflow conditions were varied by perturbing each, or both, boundary layers on the splitter plate separating the two freestream flows, upstream of shear-layer formation. The results of the chemically-reacting 'flip experiments' reveal that seemingly small changes in inflow conditions can have a significant influence not only on the large-scale structure and shear-layer growth rate, as had been documented previously, but also on molecular mixing and chemicalproduct formation, far downstream of the inflow region.

\section{Introduction}

It is an implicit assumption of turbulence and dynamic-similarity theory that, for high-enough Reynolds numbers, in the far-field regions of the flow, i.e. sufficiently far from inflow boundaries, and for times long after the initial conditions were established, the flow, at least in a statistical sense, can be characterized by local flow parameters (e.g. Batchelor 1953). In free-shear layers and some other flows, however, it is known that the inflow conditions, or 'initial' conditions in a convective frame, can exert a significant influence on the far-field properties, even at Reynolds numbers that must be considered high.

A comparison of two of the earliest turbulent 'half-jet' experiments, i.e. a shear layer for which the low-speed freestream speed is zero $\left(U_{2}=0\right)$, by Liepmann \& Laufer (1947) and Wygnanski \& Fiedler (1970), for example, revealed a significantly-larger growth rate in the latter experiment, a discrepancy eventually attributed to differences in the flow upstream of the separation (shear-layer formation) region. In the former experiment, the boundary layer was laminar, while in the latter it was turbulent. The association of this increase in shear-layer growth with the state of the splitter-plate boundary-layer (initial) condition was confirmed by the work of Batt (1975), and Oster, Wygnanski \& Fiedler (1976). Interestingly, for non-zero velocity ratios, i.e. in shear layers with a coflowing low-speed stream $\left(U_{2} \neq 0\right)$, Oster and coworkers found that a turbulent boundary layer on the high-speed splitter-plate side had the opposite effect, i.e. a reduced downstream growth rate. This behaviour for co-flowing freestreams was confirmed by the work of Browand \& Latigo (1979), Weisbrot, Einav \& Wygnanski (1982), and Mungal, Hermanson \& Dimotakis (1985). See also additional discussion and references in the review paper by Dimotakis (1991). In much 
of this work, it was found that the accompanying shear-layer velocity-fluctuation (r.m.s.) and Reynolds-stress profiles were subject to significant changes, in response to perturbed inflow conditions.

Shear layers are not unique in exhibiting such behaviour. Other examples of far-field turbulent flows that depend on inflow/initial conditions have also been documented. Wygnanski, Champagne \& Marasli (1986) reported far-field properties of turbulent wakes that depended on the shape of the body that produced the wake, e.g. airfoil, plate, etc. A sensitivity to initial conditions was also reported in the seminal work by Coles (1965) on transitional Couette-flow experiments, a behaviour corroborated by recent direct numerical simulations (Yao \& Ghosh Moulic 1995). Such a dependence of self-similar turbulence on its initial conditions is also noted by George (1989), who argues that self-similarity need not require universality, i.e. that the asymptoticallyattained flow state may depend on both inflow and initial conditions.

The requirement that the local Reynolds number must be appropriately large, if quasi-asymptotic behaviour is to be expected, stems from the need to have Reynolds stresses dominate viscous stresses, but also such that a sufficiently large range of scales and small-scale three-dimensionality can have developed and be sustainable (Dimotakis 1993). In shear layers, a relatively-well-defined transition to a state of improved mixing was documented by Konrad (1976), in gas-phase shear layers, and subsequently confirmed in liquid-phase shear layers (Breidenthal 1978; Bernal et al. 1979; Koochesfahani \& Dimotakis 1986, and subsequently others). This transition was reported to occur at the same minimum local Reynolds number, based on the local shear-layer thickness and velocity difference,

$$
R e_{\delta} \equiv \frac{\rho \Delta U \delta(x)}{\mu} \gtrsim 10^{4},
$$

or so, for both gas- and liquid-phase layers, indicating that this is not a function of the Schmidt number,

$$
S c \equiv \frac{v}{\mathscr{D}},
$$

where $\mathscr{D}$ is the species diffusivity. Similar transitions, at comparable Reynolds numbers, are documented to occur in many other flows, so this behaviour and the applicability of this minimum-Re criterion is not limited to shear layers.

This minimum-Re criterion must, however, be regarded as only necessary. Good mixing and quasi-asymptotic behaviour are documented to have additional requirements. Bradshaw (1966) indicated that shear-layer turbulence required a relatively long distance downstream to attain an asymptotic, quasi-self-similar state. In particular, on the basis of experiments in a half-jet $\left(U_{2}=0\right)$ shear layer, he proposed that such a state required a minimum downstream distance, i.e.

$$
\frac{x_{\min }}{\theta} \gtrsim 10^{3},
$$

where $x$ is the distance downstream of separation, or splitter-plate trailing edge, and $\theta$ is the momentum thickness of the separating boundary layer; an inviscid criterion in that it can, in principle, be varied independently of $R e_{\delta}$.

It was noted (Dimotakis \& Brown 1976) that such a criterion could be cast in terms of a minimum number of large-scale-structure interactions (e.g. pairings, or triplings, etc.). The proposed minimum number is, approximately, four, in terms of the estimated number of mergings,

$$
m(x)=\log _{2}\left(\frac{x}{\ell_{0}}\right) \gtrsim 4,
$$


i.e. a measure of the downstream distance, $x$, as scaled by the initial-disturbance spacing, $\ell_{0}$, with log-base- 2 in (4) assuming pairing interactions are dominant. They argued that this minimum distance and minimum number of interactions were required to amortize the effects of inflow (initial) conditions.

In subsequent work by Huang \& Ho (1990), a similar scaling of the downstream distance was proposed, in terms of a parameter

$$
P=\frac{1-r}{1+r}\left(\frac{x}{\ell_{0}}\right) \approx \frac{1-r}{1+r}\left(\frac{x}{30} \theta_{1}\right),
$$

where $r \equiv U_{2} / U_{1}$ is the shear-layer freestream velocity ratio and $\theta_{1}$ is the initial, highspeed boundary-layer momentum thickness. Huang \& Ho argued that this parameter effectively described the approach to self-similarity for a variety of shear-layer flows, i.e. for different velocity ratios, $r$ (cf. also Ho \& Huerre 1984). They suggested that a minimum value was required for self-similarity of the peak turbulence level in gas-phase flows, in particular,

$$
P_{H H} \gtrsim 8,
$$

corresponding to the location of the second large-structure pairing, according to their estimate, i.e. a criterion more relaxed than the one suggested by Dimotakis \& Brown. These authors also noted, however, that the properties of this self-similar state appeared to be influenced, if not determined, by the inflow conditions of turbulence formation, regardless of the distance from separation.

We note that the velocity-ratio scaling factor in $(5 a)$ is proportional to the local, normalized, large-scale spacing, $\ell(x) / x$, independently of the freestream density ratio (cf. Dimotakis 1986), and, therefore, as a measure, the Huang \& Ho parameter, $P$, can be seen to be proportional to $\ell(x) / \ell_{0}$, rather than $x / \ell_{0}$.

It was initially proposed by Dimotakis \& Brown (1976) and, subsequently, by others (e.g. Narayanan \& Hussain 1996) that the persistence of the effects of inflow conditions on the far field of the flow could be the result of downstream-to-upstream feedback, at least in elliptic (lab-frame subsonic) flow. In particular, if the instability response of unbounded shear layers may be classified as that of an amplifier of external disturbances (Huerre \& Monkewitz 1985), then a shear-layer developing as defined by an upstream, test-section, and outflow geometry, can be classified as a relaxation oscillator (Dimotakis \& Brown 1976), in which the flow must be understood as evolving as a global response to inflow/outflow/confinement-geometry conditions (cf. also discussion in Dimotakis 1991).

Much of the previous work included measurements of shear-layer growth rates, Reynolds stresses, etc. Only limited data are available, however, regarding the response and sensitivity of the shear-layer concentration field and its mixing properties to changes in inflow conditions. Given the large-scale effects observed in the work cited above, one might also expect significant influences in the small-scale behaviour of the flow, which is important in the molecular/diffusion-scale mixing processes. Indeed, hints of such behaviour can be found in other flows. By way of example, the far-field jet-fluid concentration-fluctuation r.m.s. statistics (a measure of unmixedness), in the far field of a turbulent jet, were found to be strongly influenced by the amplitude of small (when scaled by the jet-exit velocity), initial velocity fluctuations in the reservoir fluid that the jet discharged into (Miller 1991).

Some recent work on scalar mixing in a shear-layer flow was reported by Karasso \& Mungal (1996). These authors found the Huang \& Ho (1990) scaling parameter useful in their analysis of their measured estimates of the concentration-field probability- 
density function (p.d.f.). Dubbing $P$ (equation $(5 a)$ ) the 'pairing parameter', they proposed a higher minimum value for a converged mixed-fluid state (scalar p.d.f.) of 22 , corresponding to a distance between the average locations of third and fourth vortex interaction, more closely in accord with the original proposal of Dimotakis \& Brown (1976). Despite expressing reservations about the accuracy of their directmeasurement, laser-induced fluorescence results, on which their p.d.f. experimentalestimates relied, they suggested that this value determined the transition between a 'non-marching' p.d.f., where the most-probable concentration is nearly-independent of the transverse coordinate, $y$, and a 'tilted' p.d.f., in which the composition is correlated with the local mean, i.e. a function of $y$. In particular, they proposed that the former would be observed for $P \lesssim 22$, while the latter for $P \gtrsim 22$, i.e. that self-similarity can be expected for,

$$
P_{K M} \gtrsim 22 .
$$

Karasso \& Mungal also studied the effects of tripping the initial boundary layers, stating that the effect of tripping was to decrease the (absolute) distance, $x$, required to reach self-similarity. This is in accord with the behaviour reported by Bell \& Mehta (1990), who stated that, 'The distance required to achieve self-similarity was distinctly shorter in the tripped case, ...'. Karasso \& Mungal further suggested that, beyond this distance, inflow conditions were 'forgotten' by the turbulence, with a unique farfield configuration that only depends on the freestream-velocity and -density ratio. Since tripping the boundary layer(s) can be expected to result in an increase in the initial momentum thickness(es), it would appear that this observation is not in accord with a minimum-threshold criterion based on the pairing parameter $P$, or any other measure of distance downstream scaled by initial boundary-layer thickness scales. Finally, we note the proposal by Hussain (1977) that it is not the (thickness difference of the) laminar vs. turbulent state of the inflow boundary layers, per se, but rather the fluctuation level associated with the inflow conditions.

In the following, we present an experimental study of the effects of the inflow/initial conditions on far-field, high-Re, shear-layer mixing. In particular, the experiments were conducted at Reynolds numbers and values of distance/pairing parameters well in excess of those attained in previous work and in excess of previouslysuggested guidelines for asymptotic behaviour, to assure that this would correspond to 'fully-developed' and 'statistically self-similar' turbulence. The study employed flow visualization and 'flip experiments' (Koochesfahani, Dimotakis \& Broadwell 1985) with kinetically-fast chemical reactions, i.e. chemically-reacting flows in the high Damköhler number limit:

$$
D a \equiv \frac{\tau_{m i x}}{\tau_{\text {chem }}} \gg 1,
$$

where $\tau_{\text {mix }}$ is the fluid-mechanical molecular-mixing time and $\tau_{\text {chem }}$ is the chemicalreaction (completion) time. In this regime, chemical-product formation and heat release provides a resolution-insensitive probe of molecular (diffusion-scale) mixing, mitigating spatial-sampling/temporal-resolution difficulties of direct-measurement studies (cf. discussion in Breidenthal 1981; Koochesfahani \& Dimotakis 1986; Dimotakis 1991 and Karasso \& Mungal 1996). A detailed summary of the rationale and measures employed in the 'flip' experiment can be found in Dimotakis (1991). The brief discussion below is offered for completeness.

If the flow is of gases with a uniform (molar) heat capacity and the effects of heat release are small, a normalization of the resulting temperature-rise profile, $\Delta T(y)$, at the measuring station, $x$, by the adiabatic flame temperature rise, $\Delta T_{f}$, yields a 
dimensionless chemical-product/temperature-rise profile,

$$
\Theta(y)=\frac{\Delta T(y)}{\Delta T_{f}} .
$$

As chemical-product formation can only take place where entrained fluids are mixed on a molecular scale, this can be used to define the $1 \%$ shear-layer temperature-rise thickness, $\delta_{T}$, as the span between the two points in the profile at $1 \%$ of the peak in $\Theta(y)$. This has been found to be in good agreement with other thickness measures, such as the visual thickness, of the local shear-layer width. The $\Theta(y)$ profile is also used to compute the chemical-product thickness,

$$
\delta_{p} \equiv \int_{-\infty}^{\infty} \Theta(y) \mathrm{d} y .
$$

This represents the thickness of an equivalent layer that has risen, uniformly, to the adiabatic-flame temperature, or, equivalently, has produced chemical product by reactants mixed at the stoichiometric-mixture ratio. Finally, the product thickness is normalized by the $1 \%$ temperature-rise profile thickness, $\delta_{T}$, to obtain the dimensionless product fraction, $\delta_{p} / \delta_{T}$, and a measure of chemical-product formation that is insensitive to changes in the local shear-layer width (transverse extent).

Given the product fractions, for flows with stoichiometric-mixture ratios, $\phi=\phi_{0}$ and $\phi=1 / \phi_{0}$, for large $\phi_{0}$, the mixed-fluid fraction can estimated in the fast-kinetic regime as (Dimotakis 1991)

$$
\frac{\delta_{m}}{\delta_{T}}=\int_{-\infty}^{\infty} \mathscr{P}_{m}(y) \mathrm{d}\left(\frac{y}{\delta_{T}}\right),
$$

where

$$
\mathscr{P}_{m}(y) \simeq \int_{\epsilon_{\phi_{0}}}^{1-\epsilon_{\phi_{0}}} p(X, y) \mathrm{d} X,
$$

where $X$ is the high-speed freestream-fluid mole fraction, and $p(X, y)$ is its probability density function (p.d.f.) at the transverse coordinate, $y$, at the measuring station. This can be estimated from the measured product thicknesses from a flip experiment, i.e.

$$
\frac{\delta_{m}}{\delta_{T}} \simeq \frac{1}{1+1 / \phi_{0}}\left[\left(\frac{\delta_{p}}{\delta_{T}}\right)_{\phi_{0}}+\left(\frac{\delta_{p}}{\delta_{T}}\right)_{1 / \phi_{0}}\right],
$$

where

$$
\epsilon_{\phi_{0}} \simeq \frac{1}{2\left(1+\phi_{0}\right)}, \quad \text { e.g. } \quad \epsilon_{8} \simeq 0.056
$$

For shear layers in the kinetically-fast regime, $\delta_{m} / \delta_{T}$ provides a spatial/temporal resolution-insensitive measurement of the molecularly-mixed fluid fraction at the measurement location. In practice, that the kinetically-fast regime has been attained can be ascertained by chemical product formation that remains unaltered as the kinetic rate of the chemical reaction is varied. In this regime, chemical-product formation is at high $D a$, i.e. mixing-rate-limited, rather than kinetic-rate-limited.

\section{Experiments}

The experiments reported here were conducted in the GALCIT Supersonic Shear Layer $\left(S^{3} \mathrm{~L}\right)$ facility (Hall \& Dimotakis 1989; Hall 1991). This is a two-stream, blow- 


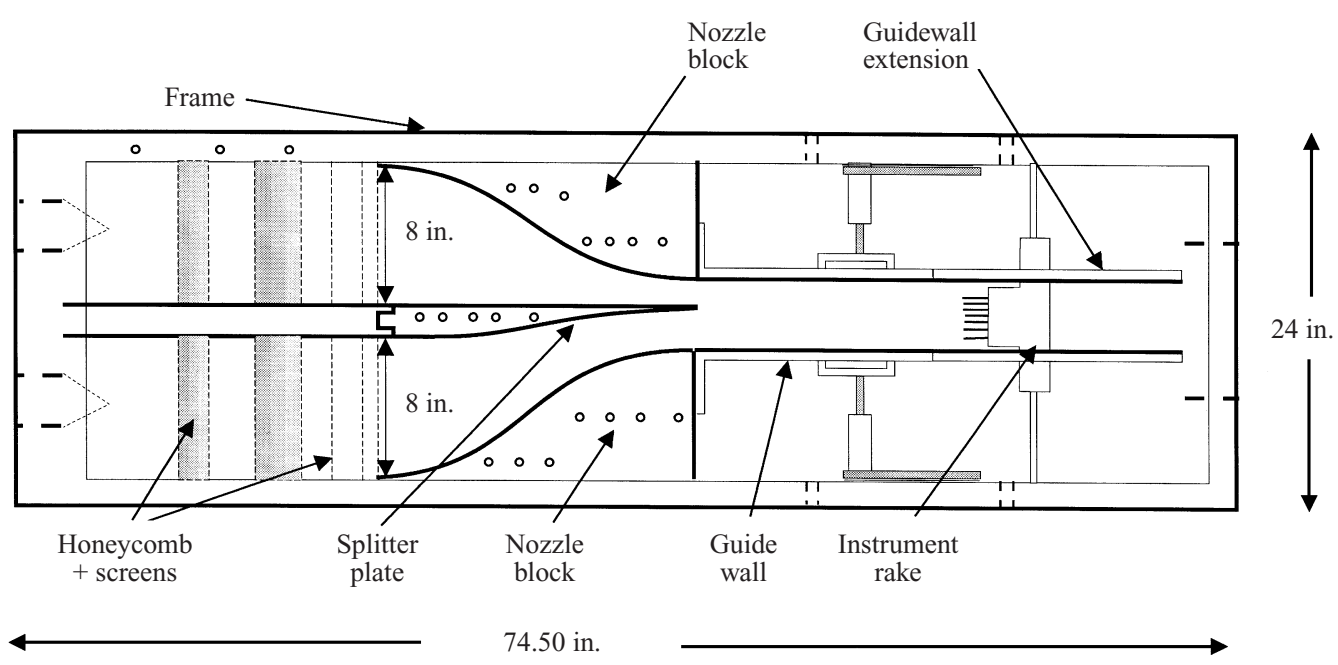

FIGURE 1. Shear-layer flow test-section schematic.

down, wind tunnel, nominally operating at atmospheric (static) pressure, with a run time of $t_{\text {run }}>t_{d a t} \simeq 2-4 \mathrm{~s}$, where $t_{d a t}$ is the data-recording time. Gas for the two freestreams is supplied by independent flow systems.

The $\mathrm{S}^{3} \mathrm{~L}$ facility can accommodate flows with fast-kinetic reactants, whose chemical reaction and associated temperature rise in a known heat-capacity stream yields measurements of molecular mixing in the turbulent shear-layer mixing region where fluid from the two freestreams is entrained and mixed. For the experiments reported here, a dilute mixture of $\mathrm{H}_{2}$ and $\mathrm{NO}$ is carried in the high-speed freestream and a dilute concentration of $F_{2}$ is carried in the low-speed freestream. The remaining freestream fluid diluent components are selected to maintain a nominal density match and uniform molar heat-capacity fluid, as reactant compositions are varied to produce the desired stoichiometric-mixture ratios. A schematic of the test section is shown in figure 1.

For the subsonic shear-layer flows investigated in these experiments, the high-speed stream speed is maintained by controlling pressure in the high-speed stream plenum by means of an active-control system, operated in an open-loop (program-control) configuration. Gas to this plenum is supplied from a $1.2 \mathrm{~m}^{3}$ pressure vessel, at a pressure that ranges from $2400 \mathrm{kPa}$ to $2100 \mathrm{kPa}$ during a blowdown run. The flow is maintained isothermal by means of the large heat capacity of a rolled aluminium screen that nearly fills the interior pressure-vessel space, occupying, roughly, $15 \%$ of the volume. The pressure in the low-speed freestream supply plenum is set by a choked metering valve, adjusted and kept fixed during each run. Gas to this plenum is supplied from a $0.57 \mathrm{~m}^{3}$ vessel, containing a bladder bag with the fluorine charge and pressurized, in turn, by a $12.7 \mathrm{~m}^{3}$ surge tank whose pressure falls by $3-5 \%$ during the run time, $t_{r u n}$, and less during the data-recording time interval, $t_{\text {dat }}$.

Coarse-mesh-screen, honeycomb, and a series of fine-mesh-screen sections are used for turbulence management in each plenum. The two streams are separated in their respective plena by a splitter plate, which is flat on the top (high-speed) surface and curved on the bottom (low-speed) surface, as shown in figure 2. Surface finish quality was estimated to have an (average) roughness of $0.4 \mu \mathrm{m}$, using General Electric surface-finish sample gauges. The splitter plate has a trailing-edge thickness of $0.13 \mathrm{~mm}$. 


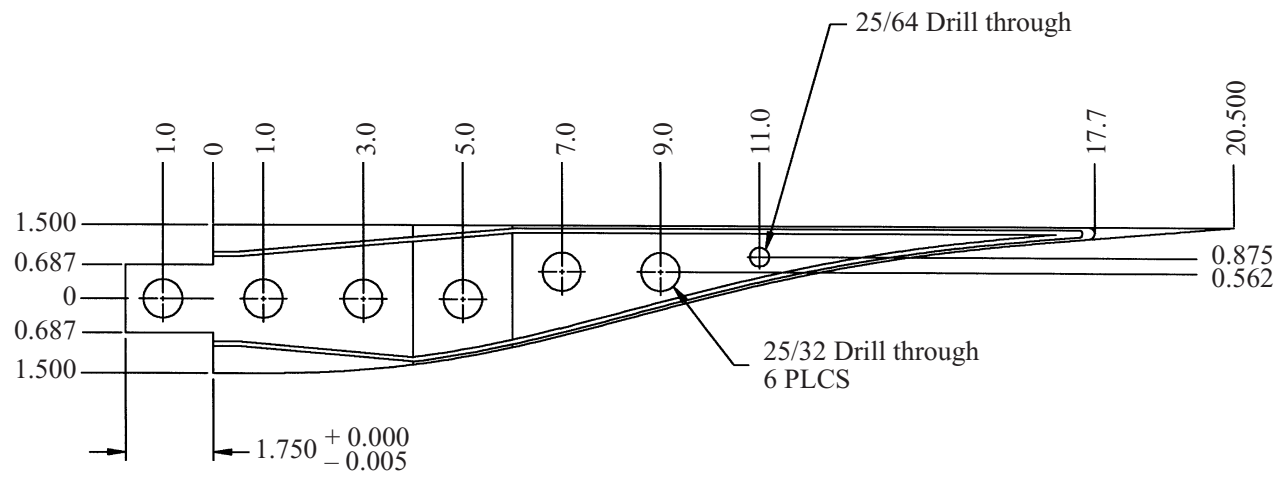

FIGURE 2. Drawing of the splitter plate used in these experiments (figure 1).

Dimensions in English units.

The flow in the test section is confined by the top and bottom guidewalls, with parallel optical windows serving as sidewalls, with a span, $b=15.3 \mathrm{~cm}$. The upper guidewall is maintained horizontal (parallel to top splitter-plate surface), with the lower guidewall adjusted, as necessary in each flow, to remove mean streamwise pressure gradients. This arrangement yields a shear layer with negligible pressuregradient and transverse bounding-wall effects.

The shear-layer experiments were performed at a single nominal flow condition, with freestream speeds

$$
U_{1} \simeq 100 \mathrm{~m} \mathrm{~s}^{-1} \quad \text { and } \quad U_{2} \simeq 40 \mathrm{~m} \mathrm{~s}^{-1}, \quad \text { i.e. } \quad r \equiv \frac{U_{2}}{U_{1}} \simeq 0.4,
$$

and a density ratio of

$$
s \equiv \frac{\rho_{2}}{\rho_{1}} \simeq 1.0 .
$$

The speed and density ratios were chosen to facilitate a comparison with previous work on chemically-reacting flows, at similar freestream conditions (e.g. Mungal et al. 1985; Frieler \& Dimotakis 1988; Hermanson \& Dimotakis 1989; Bond \& Dimotakis 1996, as well as Island 1997), who investigated molecular mixing in shear layers at different conditions, however, using a fluorescence-quenching ('cold-chemistry') technique.

The pressure and temperature profiles in the present experiments were recorded at a single measuring station, $x=0.365 \mathrm{~m}$, where $x$ is measured from the location of the splitter-plate tip. Profiles were measured as a function of the transverse coordinate, $y$, that is also referenced to the splitter-plate tip. The resulting shear layers have a local Reynolds number at the measuring station in the range

$$
1.8 \times 10^{5} \lesssim \operatorname{Re}_{\delta} \equiv \frac{\rho \Delta U \delta_{T}(x)}{\mu} \lesssim 2.4 \times 10^{5},
$$

where $\Delta U \equiv U_{1}-U_{2}$ is the freestream velocity difference, and $\rho$ and $\mu$ are the mixedfluid density and viscosity, respectively. These are calculated using the properties of a gas mixture with a high-speed fluid mole fraction given by $X_{E}$, which reflects the mole ratio, $E_{n}$, of fluid entrained from the two freestreams, at the large scales of the flow (Dimotakis 1986), i.e.

$$
X_{E} \simeq \frac{E_{n}}{E_{n}+1}
$$




$\begin{array}{lcccc} & \theta(\mathrm{mm}) & R e_{\theta} \equiv \rho U \theta / \mu & x / \theta & P \text { (equation }(5 a) \text { ) } \\ \text { High-speed side: } & 0.11 & 640 & 3.3 \times 10^{3} & 47 \\ \text { Low-speed side: } & 0.17 & 400 & 2.1 \times 10^{3} & -\end{array}$

TABLE 1. Untripped (natural) splitter plate boundary-layer flows.

Case 1. Fluorine-rich $\left(\phi=8, \Delta T_{f} \simeq 171 \mathrm{~K}\right)$ :

$U_{1} \simeq 100 \mathrm{~m} \mathrm{~s}^{-1}: \quad 8.00 \% \mathrm{Ar}, 15.07 \% \mathrm{He}, 0.93 \% \mathrm{H}_{2}, 0.14 \% \mathrm{NO}, 75.86 \% \mathrm{~N}_{2}$ $U_{2} \simeq 40 \mathrm{~m} \mathrm{~s}^{-1}: \quad 16.00 \% \mathrm{He}, 8.00 \% \mathrm{~F}_{2}, 76.00 \% \mathrm{~N}_{2}$.

Case 2. Hydrogen-rich $\left(\phi=1 / 8, \Delta T_{f} \simeq 267 \mathrm{~K}\right)$ :

$U_{1} \simeq 100 \mathrm{~m} \mathrm{~s}^{-1}: \quad 8.00 \% \mathrm{Ar}, 4.00 \% \mathrm{He}, 12.00 \% \mathrm{H}_{2}, 0.75 \% \mathrm{NO}, 75.25 \% \mathrm{~N}_{2}$

$U_{2} \simeq 40 \mathrm{~m} \mathrm{~s}^{-1}: \quad 6.45 \% \mathrm{Ar}, 16.00 \% \mathrm{He}, 1.55 \% \mathrm{~F}_{2}, 76.00 \% \mathrm{~N}_{2}$

Case 3. Hydrogen-rich, reduced concentration $\left(\phi=1 / 8, \Delta T_{f} \simeq 171 \mathrm{~K}\right)$ :

$U_{1} \simeq 100 \mathrm{~m} \mathrm{~s}^{-1}: \quad 8.00 \% \mathrm{Ar}, 8.00 \% \mathrm{He}, 8.00 \% \mathrm{H}_{2}, 0.50 \% \mathrm{NO}, 75.50 \% \mathrm{~N}_{2}$

$U_{2} \simeq 40 \mathrm{~m} \mathrm{~s}^{-1}: \quad 6.97 \% \mathrm{Ar}, 16.00 \% \mathrm{He}, 1.03 \% \mathrm{~F}_{2}, 76.00 \% \mathrm{~N}_{2}$

Case 4. Non-reacting:

$U_{1} \simeq 100 \mathrm{~m} \mathrm{~s}^{-1}: \quad 8.00 \% \mathrm{Ar}, 16.00 \% \mathrm{He}, 76.00 \% \mathrm{~N}_{2}$

$U_{2} \simeq 40 \mathrm{~m} \mathrm{~s}^{-1}: \quad 8.00 \% \mathrm{Ar}, 16.00 \% \mathrm{He}, 76.00 \% \mathrm{~N}_{2}$

TABLE 2. Freestream speeds and compositions.

assuming conservation of (total) enthalpy to estimate the mixed-fluid density, $\rho$, and the method of Wilke (1950) to estimate the mixed-fluid (shear) viscosity, $\mu$. These parameters were kept nominally fixed to isolate the effects of variable-inflow conditions, as each is known to influence shear-layer growth, entrainment, and mixing. The range in (11) arises from variations in composition as well as (primarily) the local shear-layer thickness, $\delta(x)$, in each case, as will be documented below.

Changes in inflow conditions were effected through various combinations of trip wires on the splitter plate. For the freestream conditions of these experiments, estimated boundary-layer properties at the splitter-plate tip are listed in table 1. For each condition, three sets of experimental runs were performed to create a 'flip experiment'. These relied on chemically-reacting flows, with stoichiometric-mixture ratios of $\phi=\phi_{0}$ and $\phi=1 / \phi_{0}$, for $\phi_{0}=8$, in addition to non-reacting flows ('cold' runs). Table 2 lists the flow conditions and freestream fluid compositions for these runs.

Local Reynolds numbers for the shear layers investigated, at the $x=0.365 \mathrm{~m}$ measuring station (equation (11)), are higher than in the previous shear-layer, molecularmixing work by Mungal et al. (1985), which included an exploratory investigation of inflow effects. In the upgraded facility used for the present experiments, it was possible to increase flow speeds (and measuring-station Reynolds numbers), such that the natural (unperturbed) flow boundary layers could be expected to remain laminar, but at high-enough Reynolds numbers to permit a transition to turbulent boundary layers, if tripped. For higher Reynolds numbers yet, the naturally-occurring splitter-plate boundary layers would be transitional, or naturally turbulent, and purposely altering inflow conditions by tripping would not be expected to have as large a (reproducible) effect.

The values of the adiabatic-flame temperature rise, $\Delta T_{f}$, for these runs are all in a regime where dilatation and other heat-release effects have been documented to be small (Hermanson \& Dimotakis 1989). As was noted in those investigations, the 
main heat-release effect, at the values of $\Delta T_{f} / T_{0}$ in the present experiments, where $T_{0}$ is the (common) stagnation temperature of the two freestreams, is the possible dilatation-induced flow acceleration in the confined-flow environment. In the present experiments, however, such effects were accommodated by adjusting the bottom (lowspeed) guidewall to maintain a zero streamwise pressure gradient (no acceleration), for all cases, simulating an unbounded shear-layer flow, as noted above. That heatrelease effects are small in the present experiments can also be confirmed, directly, by comparing the $1 \%$ thicknesses, $\delta_{T}$, of Case 1 runs with those of Case 2 runs, for which $\Delta T_{f} / T_{0}$ is substantially different, as well as the comparison of Case 2 with Case 3 runs, if both are in the fast-kinetic regime.

Separate experiments were performed to ensure that these were in the fast chemicalkinetic limit, i.e. at high Damköhler numbers (equation (6)), by proportionally reducing the concentrations of all reactants. This decreases the kinetic rate by both decreasing the number density of the reactant species as well as the adiabatic temperature rise and associated Arrhenius kinetics constants, i.e. an increase in $\tau_{c h e m}$, holding $\tau_{\text {mix }}$ constant.

Three types of experimental results will be documented here: colour-schlieren visualizations, mean-temperature measurements, and mean-velocity measurements. Schlieren visualization records the instantaneous, overall, spanwise-averaged (twodimensional) structure of the layer. This indicates whether the shear-layer growth is linear and also, depending on the selected sensitivity, whether any significant acoustic disturbances are present. Colour schlieren, as discussed by various authors (e.g. Settles 1985), provides information on both components of the line-of-sight-averaged indexof-refraction gradients in the flow, as opposed to a single component recorded by traditional (black-and-white), knife-edge systems.

The temperature profiles are recorded by a rake of sixteen, $50 \mu \mathrm{m}$ diameter, chromel/alumel, exposed-junction thermocouples, that effectively produce running temporal averages of the temperature at the respective measurement locations. Their response (averaging) time is estimated to be approximately $20 \mathrm{~ms}$. The velocity data were deduced from direct Pitot- and static-pressure measurements, as described in Hermanson \& Dimotakis (1989) and below. As with the temperature data, the velocity data represent running temporal averages at the measurement station.

For each flow condition, the corresponding non-reacting-flow temperature profile is subtracted to compute the mean temperature-rise measurements, $\Delta T_{i}=\Delta T\left(x, y_{i}\right)$, at each probe location. These are divided by the adiabatic flame temperature rise at the stoichiometric-mixture ratio, $\Delta T_{f}$, yielding the normalized mean temperature-rise measurements, $\Theta_{i}=\Delta T_{i} / \Delta T_{f}$. These are least-squares fit with a functional form

$$
\Theta(y) \equiv \frac{\Delta T(y)}{\Delta T_{f}}=\exp \left[-\left(p_{0}+p_{1} y+p_{2} y^{2}+p_{3} y^{3}+p_{4} y^{4}\right)\right] .
$$

This analytical expression is used to estimate the $1 \%$ profile thickness, $\delta_{T}$, as used in the definition of the local Reynolds number (11), and the mixed-fluid fraction, $\delta_{m} / \delta_{T}$, as outlined above.

Velocity profiles are calculated from the Pitot pressure measurements in the usual manner,

$$
U_{i}=\left(\frac{2\left[P_{p}\left(y_{i}\right)-P_{g}\right]}{\rho\left(y_{i}\right)}\right)^{1 / 2},
$$

with the $P_{p}\left(y_{i}\right)$ the Pitot pressure measurements at the probe locations, $y_{i}, P_{g}$ the 


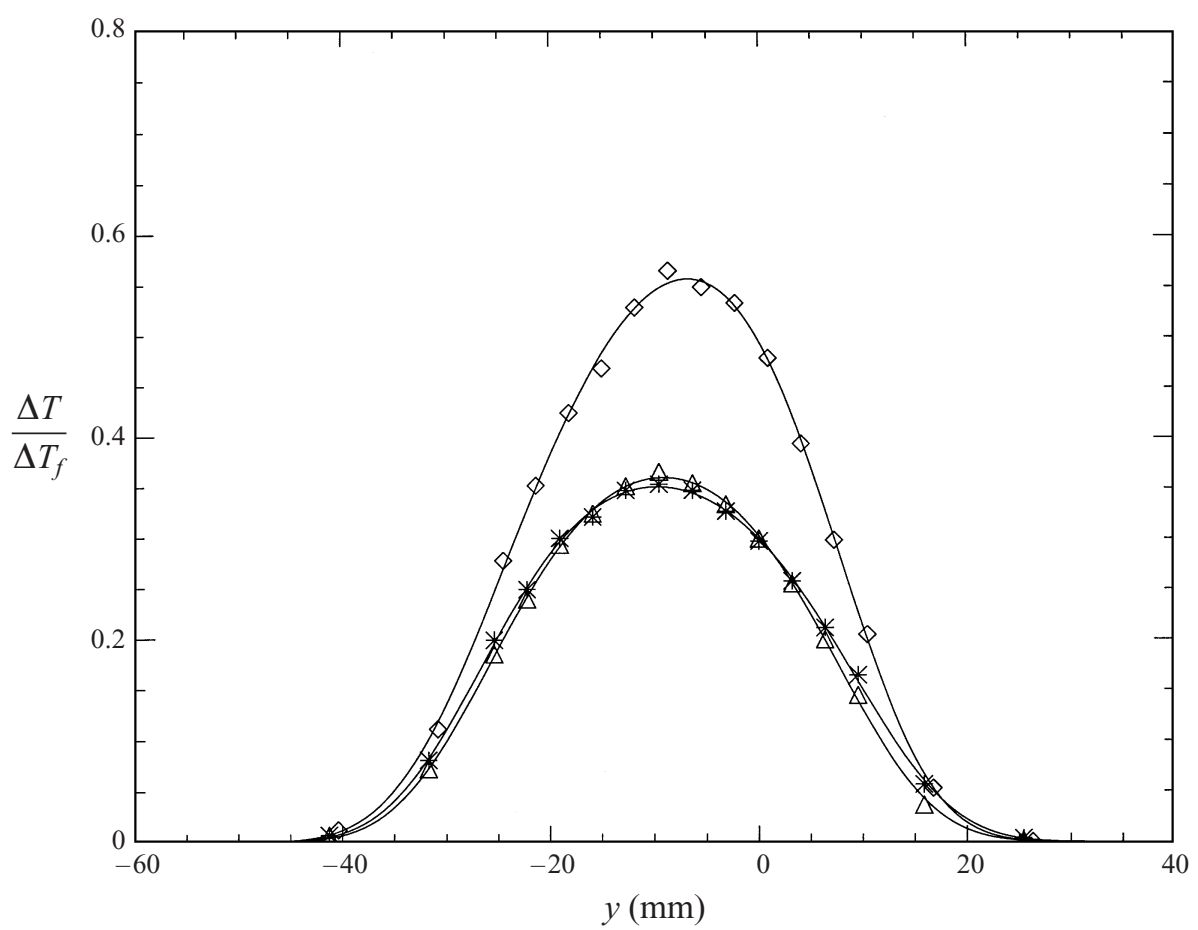

FIGURE 3. Normalized temperature-rise data with natural (untripped) boundary layers. Diamonds: Case $1, \phi=8$. Triangles: Case 2, $\phi=1 / 8$. Asterisks: Case $3, \phi=1 / 8$, reduced chemical-kinetic rate.

guidewall (static) pressure measurement, and $\rho\left(y_{i}\right)$ the local density. These are then least-squares fit with an analytical form,

$$
U(y)=A+B \tanh \left(q_{0}+q_{1} y+q_{2} y^{2}+q_{3} y^{3}\right) .
$$

This provides measurements of the freestream speeds, $U_{1}$ and $U_{2}$, and contributes to general monitoring of each run. For these incompressible flows, the density, $\rho(y)$, is estimated using the freestream temperature, $T_{0}$, and density $\rho_{0}$, and evaluating the temperature-rise profile (13) at each probe location, i.e.

$$
\rho(y) \simeq \frac{\rho_{0}}{1+\left[\Delta T_{f} / T_{0}\right] \Theta(y)} .
$$

The data-recording time, $t_{d a t} \simeq 2-4 \mathrm{~s}$, in these experiments corresponds to the passage of at least $10^{3}$ large-scale structures, i.e. $t_{d a t} \simeq U_{c} / \ell(x) \gtrsim 10^{3}$, where $U_{c} \simeq$ $\left(U_{1}+U_{2}\right) / 2 \simeq 70 \mathrm{~m} \mathrm{~s}^{-1}$ is the large-scale structure convection speed (unity densityratio freestreams) and $\ell(x)$ is the large-scale-structure spacing, at the measuring station.

\section{Results and discussion}

Normalized temperature-rise profile measurements for the natural (untripped) flow conditions (Case 1) are plotted in figure 3. The corresponding velocity-profile data are plotted in figure 4.

To ensure that the flows were in the kinetically-fast, high $D a$ limit (cf. (6) and associated discussion), reactant concentrations for the kinetically slower of the two 


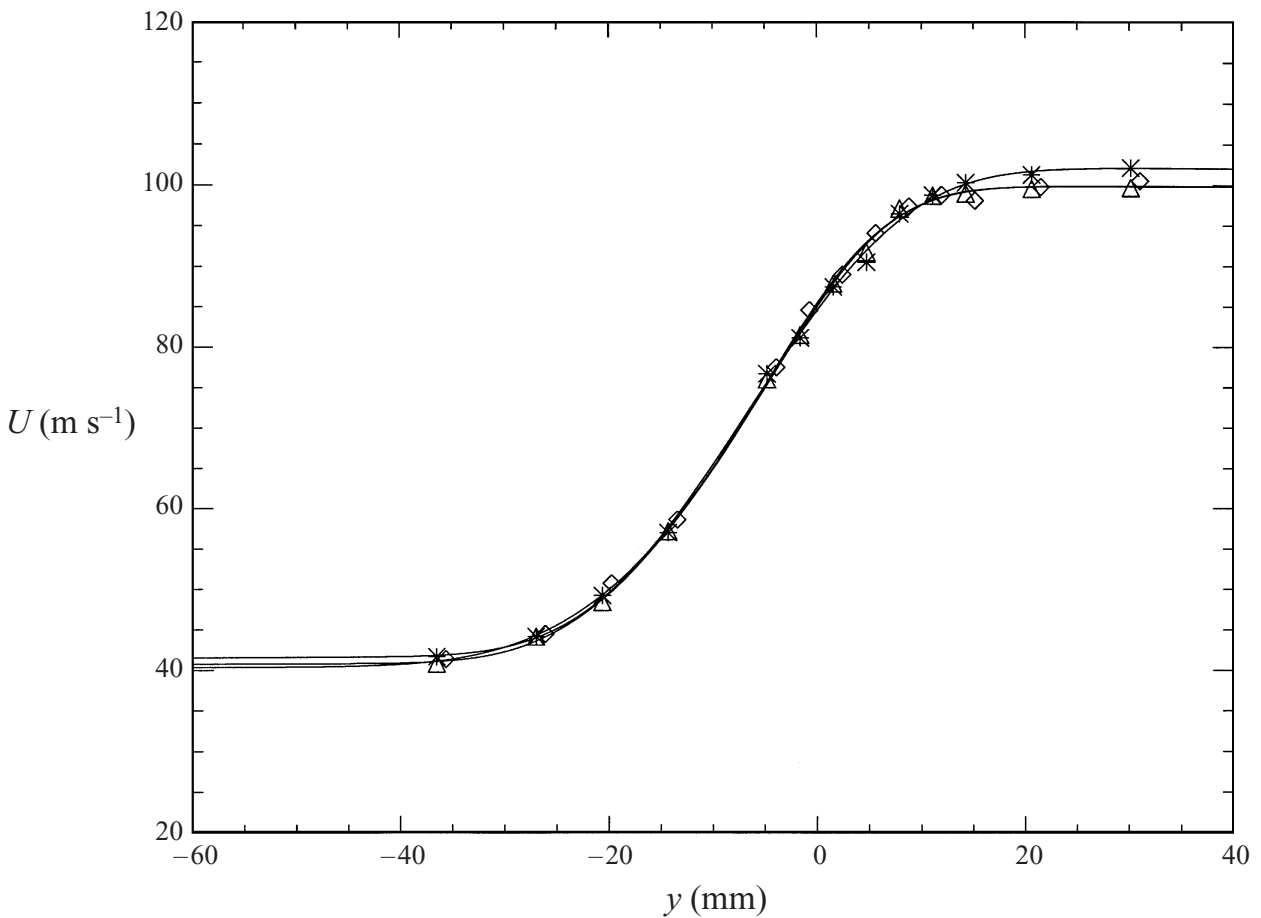

FIGURE 4. Velocity profiles for natural (untripped) flows. Legend as in figure 3.

cases $\left(\mathrm{H}_{2}\right.$-rich, $\phi=1 / 8$ case) were reduced to those listed under Case 3 (table 2). Damköhler numbers associated with the $\mathrm{F}_{2}$-rich $(\phi=8)$ case of the flip experiment are higher than those of the $\mathrm{H}_{2}$-rich $(\phi=1 / 8)$ case, with higher concentrations of the rate-limiting (free) fluorine atoms in the former case. The Damköhler number ratio for the two $\phi=1 / 8$ cases is computed to be larger than 3 , if a homogeneouscomposition (well-stirred), constant-pressure, 'balloon' reactor (Dimotakis \& Hall 1987 ) is used to estimate the reaction-completion times: $\tau_{\text {chem }}=0.45 \mathrm{~ms}$ and $1.50 \mathrm{~ms}$, respectively (cf. (6)). As can be seen in figure 3 , the two $\phi=1 / 8$ temperature-rise profiles, corresponding to the already kinetically slower of the two $\phi$, are within typical experimental reproducibility, demonstrating that the flows are in the high $D a$ regime. The profile collapse also demonstrates that heat-release effects are weak; the adiabatic flame temperature rise, $\Delta T_{f}$, was lower by over a factor of 1.5 for the kinetically-slower case (cf. table 2). No significant heat-release effects are manifest in the velocity profiles, with differences typical of the small run-to-run variations. The smooth curves in figure 4 are plots of the corresponding analytical fits (14b).

Acoustic forcing is known to exert an important influence on both large-scale entrainment (e.g. Koochesfahani \& MacKinnon 1991), as well as on turbulent-fluctuation profiles (e.g. Wygnanski, Oster \& Fiedler 1979). Acoustic forcing of the type that would resonate with some local, large-scale passage frequency at an intermediate downstream location would result in nonlinear shear-layer growth rates (e.g. Wygnanski et al. 1979; Oster \& Wygnanski 1982; Ho \& Huang 1982; Ho \& Huerre 1984; Dziomba \& Fiedler 1985; Roberts 1985 and Roberts \& Roshko 1985).

All shear layers investigated in the present experiments exhibit a very-nearly-linear growth rate, i.e. $\delta_{v i s}(x) \propto x$, as seen in the flow-visualization data reproduced in figure 5. Such data represent instantaneous flow visualizations, i.e. are not time 

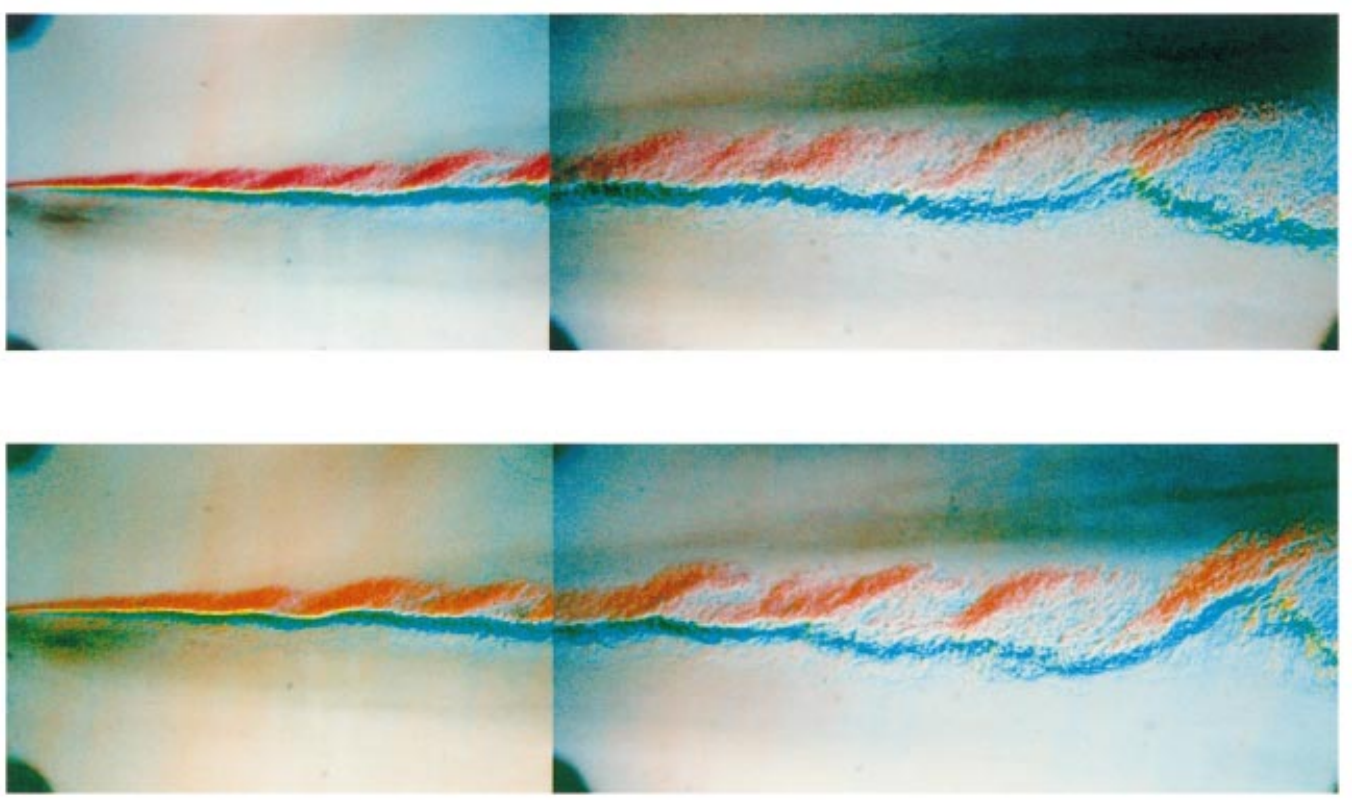

FIGURE 5. Composite colour-schlieren images of untipped (natural), Case 2 flows. Note linear growth and similar large-scale structure, indicating self-similar flow.


FIGURE 8. Composite colour-schlieren images of tipped ( $0.8 \mathrm{~mm}$ wire on high-speed side), Case 2 flows. Note linear growth and geometrically-similar large-scale structures. 
averaged. They are subject, however, to an evolving aspect ratio, $b / \delta(x)$, where $b=15.3 \mathrm{~cm}$ is the fixed span, while $\delta(x) \propto x$, as the layer grows. Any threedimensional structure of the flow will be scaled by the linearly-increasing local width, $\delta(x)$, at its largest scales. As a consequence, an apparent evolution in 'coherence', i.e. an artifact of the visualization, can be expected in such spanwise-averaged measurements that makes the flow look as if it is becoming increasingly two-dimensional with increasing downstream distance.

The temperature-rise data in figure 3 were used to compute the various quantities described above. In particular, for this natural (untripped) flow,

$$
\left.\begin{array}{llll}
\text { Case 1: } & \phi=8, & \delta_{T} / x=0.178, & \delta_{p} / \delta_{T}=0.277 ; \\
\text { Case 2: } & \phi=1 / 8, & \delta_{T} / x=0.177, & \delta_{p} / \delta_{T}=0.184,
\end{array}\right\}
$$

where $\delta_{T}$ is the temperature-rise profile $1 \%$ thickness and $\delta_{p}$ is the product thickness (8). These numbers are determined with a precision for each flow of $\lesssim 1 \%$, with a run-to-run reproducibility, at these flow conditions, in the range of $1 \%-3 \%$. The reproducibility of the local shear layer width, $\delta_{T} / x$, between different runs is indicative of this behaviour. These can be used to compute the mixed-fluid fraction $(9 a)$,

$$
\delta_{m} / \delta_{T}=0.410 .
$$

As can be seen in figure 3, the locations of the peak temperature rise are nearly aligned, despite the extreme shift in stoichiometric-mixture ratio (from $\phi=1 / 8$ to $\phi=8$ ). Such behaviour is indicative of a 'non-marching' p.d.f., i.e. a p.d.f. that is nearly independent of $y$, corresponding to compositions that do not favour fluid entrained from the nearest freestream. These profiles were recorded in a high local Reynolds number $\left(R e_{\delta} \sim 2 \times 10^{5}\right)$ shear-layer flow, far downstream $\left(x / \theta_{1} \simeq 3.3 \times 10^{3}\right)$, and corresponding to a high value of the pairing parameter $(P \simeq 47)$. As discussed in the Introduction, by any of the measures employed to characterize such flows (cf. (1), (3), (4) and $(5 a-c))$, these data were recorded in an environment that must be regarded as representing fully-developed turbulent shear flow.

The implied mixed-fluid composition at these conditions is at variance with the report by Karasso \& Mungal (1996). As discussed by these authors, one might expect some spatial 'flip' shift in the two temperature-rise peaks, i.e. that the $\mathrm{F}_{2}$-rich $(\phi=8)$ case would have a peak temperature shifted towards the lean-reactant, $\mathrm{H}_{2}$-bearing freestream, and vice versa for the $\mathrm{H}_{2}$-rich $(\phi=1 / 8)$ case, at least in high Reynolds number shear-layer flows. Examples of this behaviour are found in the references cited above (cf. data and discussion in Dimotakis 1991), and are indicative of a probabilitydensity function (p.d.f.) that is 'marching', as it has become known, in which mixture composition favours the fluid of the closer of the two freestreams and is a weak function of the transverse coordinate, $y$. A similar absence of flip shift was observed in the lower-Re, liquid-phase, chemically-reacting shear-layer flows of Koochesfahani \& Dimotakis (1986). Such behaviour was attributed to Schmidt number effects, longer Lagrangian-time-frame delays to mix, or finite chemical-kinetic rate effects in discussions of the BBM model (cf. Broadwell \& Mungal 1991). Rogers \& Moser $(1992,1994)$ have attributed this to a higher degree of two-dimensional organization in the flow. In their simulations of temporally-developing shear layers, these authors found a correlation between the degree of two-dimensionality of conditions used to initialize the simulation and the characteristics of the p.d.f. that persists for the duration of the simulation time. In particular, for initial conditions dominated by twodimensional disturbances, the flow structure largely maintained the two-dimensionality 


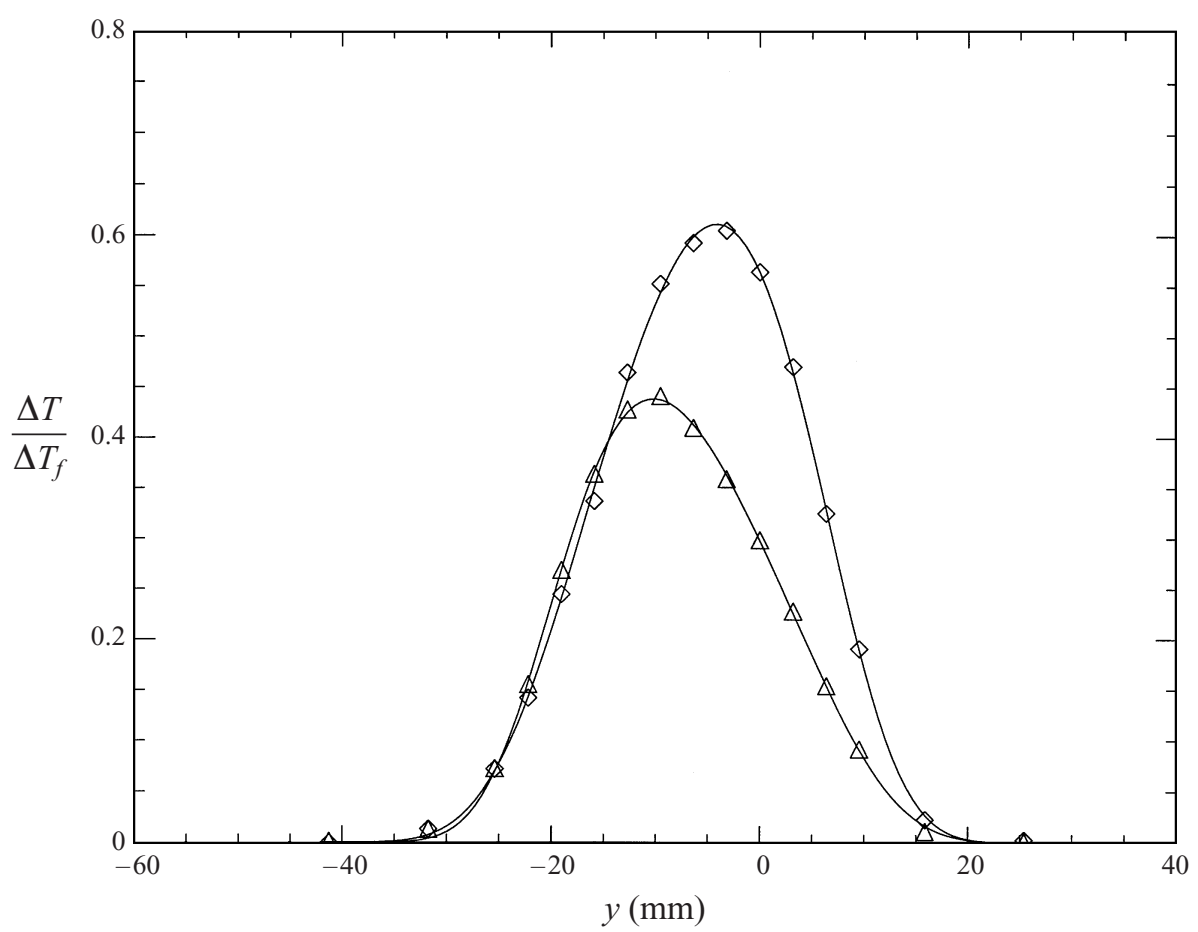

FIGURE 6. Normalized temperature-rise data with tripped high-speed boundary layers. Diamonds: Case $1(\phi=8)$. Triangles: Case $2(\phi=1 / 8)$.

yielding a scalar field described by a non-marching p.d.f. When initialized by a more three-dimensional disturbance field, however, the flow evolved differently, yielding a marching p.d.f. These temporal-flow simulations were conducted at $R e_{\delta} \lesssim 2 \times 10^{4}$, i.e. just past the critical mixing transition (1). As noted by the authors, the strongly-twodimensional flow reached only an approximately self-similar state by the end of the simulation.

To explore the sensitivity to inflow conditions, a $0.8 \mathrm{~mm}$ diameter trip wire was placed on the upper (high-speed) face of the splitter plate, $50 \mathrm{~mm}$ upstream of the splitter-plate trailing edge. The naturally-occurring boundary layer is laminar, with a momentum thickness of $0.095 \mathrm{~mm}$ at the trip location (cf. with trailing edge values in table 1), estimated using the method of Thwaites. The trip-wire size and position matched those employed by Mungal et al. (1985), in the previous (subsonic) version of this facility. White (1974) recommends a Reynolds number based on wire diameter in excess of 850 to ensure transition (in a receptive boundary layer). The trip-wire Reynolds number in this flow is estimated to be $4.6 \times 10^{3}$. The resulting temperaturerise and streamwise-velocity profile data, corresponding to those plotted in figures 3 and 4 , are plotted in figures 6 and 7, respectively, with schlieren data reproduced in figure 8 (see page 126).

The temperature-rise profiles in figure 6 are measured $0.415 \mathrm{~m}$, or approximately 500 trip-wire diameters, downstream of the trip location, in the 'far-field' of this perturbation. The untripped flow, at the same location, satisfies all previously-proposed criteria for fully-developed flow. In the case of the tripped boundary-layer flow, even if the boundary layer (momentum thickness) was assumed to increase to $1 / 3$ the trip-wire diameter, the same criteria would still be satisfied. 


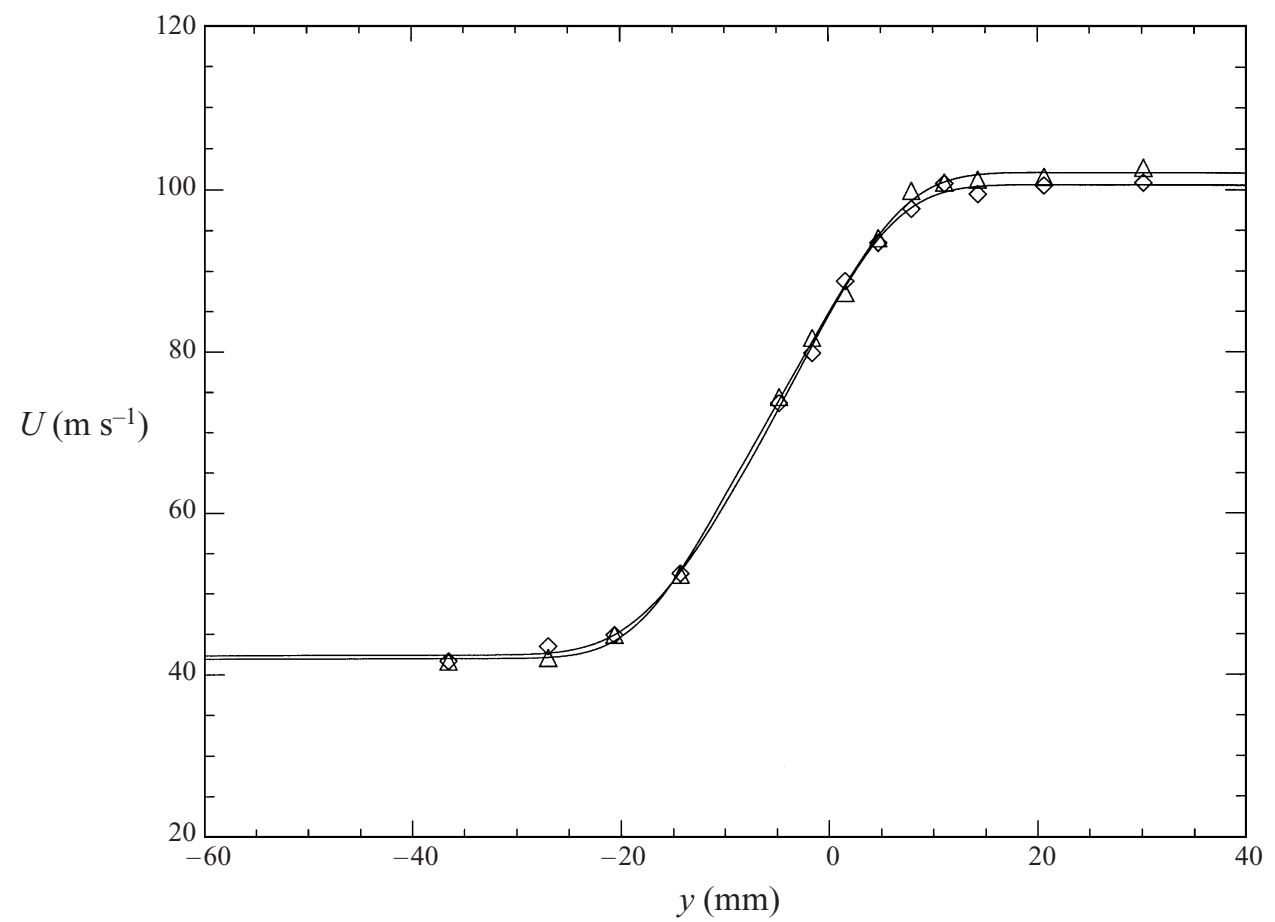

FIGURE 7. Velocity profiles for the flows with tripped high-speed side boundary layers. Legend as figure 6.

The shear-layer growth and mixed-fluid quantities, for the flows with the high-speed boundary-layer tripped, were also derived from these temperature-rise profile data,

$$
\begin{aligned}
& \text { Case 1: } \left.\quad \phi=8, \quad \delta_{T} / x=0.140, \quad \delta_{p} / \delta_{T}=0.293 ;\right\} \\
& \text { Case 2: } \left.\quad \phi=1 / 8, \quad \delta_{T} / x=0.138, \quad \delta_{p} / \delta_{T}=0.211,\right\}
\end{aligned}
$$

yielding a mixed-fluid fraction of

$$
\delta_{m} / \delta_{T}=0.448 .
$$

Comparing with the untripped boundary-layer data (16a), tripping the high-speed boundary layer can be seen to have resulted in a growth rate, $\delta_{T}(x) / x$, that is lower by $21 \% \pm 1.5 \%$. This must be regarded as a substantial reduction considering that this flow should have attained quasi-asymptotic behaviour, i.e. one in which inflow/initial conditions are expected to exert a minimal influence, if any. This reduction, however, is in qualitative accord with previous investigations, at lower $R e_{\delta}$, of two-stream (as opposed to half-jet) shear layers, as discussed in the Introduction.

Significantly, the temperature-rise data for the tripped high-speed boundary-layer exhibit a 'flip' shift, indicating that a transition to a 'tilted' molecularly-mixed-fluid p.d.f. has occurred, i.e. one registering that the mixed-fluid composition is now a function of the transverse coordinate, $y$. If this is to be expected of turbulence in a 'more-developed' state, as suggested by Karasso \& Mungal (1996), it cannot be attributed to an increase in Reynolds number. The $21 \%$ reduction in local thickness, $\delta_{T}(x)$, induced by tripping the high-speed side boundary layer, decreases the local Reynolds number, $R e_{\delta}$, by the same amount. The data are also at variance with the proposed Karasso \& Mungal criteria for p.d.f. evolution and behaviour, in terms of 


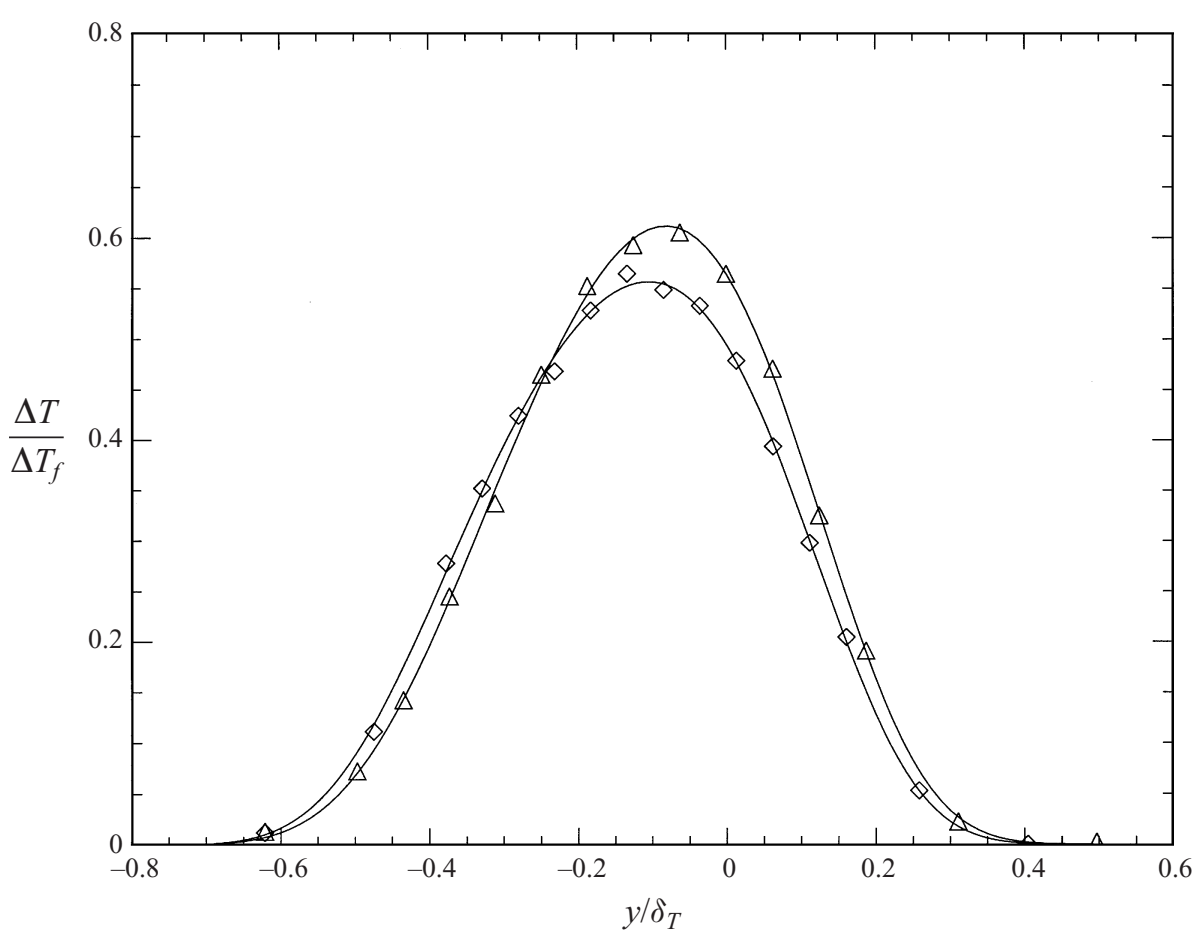

FIGURE 9. Normalized temperature-rise data for tripped and untripped splitter-plate boundary layers at $\phi=8$. Transverse coordinate normalized by $\delta_{T}$. Triangles: tripped high-speed side; diamonds: untripped high-speed side.

the change in $x / \theta_{1}$. The tripped flow has a slightly-thicker high-speed boundary layer, i.e. a higher $\theta_{1}$, and, therefore, a lower scaled downstream distance, $x / \theta_{1}$, as well as pairing parameter, $P$. Changes in this direction should have had the opposite effect, i.e. taken a 'non-marching' p.d.f., characteristic of 'undeveloped' flow, to a 'marching' p.d.f., characteristic of 'developed' flow, according to these authors.

Tripping reduced entrainment and shear-layer growth far downstream of the change in inflow conditions. Interestingly, it also produced more vigorous stirring and effective molecular mixing. The latter can be ascertained by plotting the data, scaling the transverse coordinate by the local width, i.e. $y / \delta_{T}$, as in figures 9 and 10 . It is in these units that the computation of the mixed-fluid fraction, $\delta_{m} / \delta_{T}$, is performed (equation (9)). The increase in chemical product and molecular mixing within the shear layer, in each case, is clear. As can be seen by comparing the product fractions, $\delta_{p} / \delta_{T}$, for the two cases, (16) vs. (17), the increase is approximately $6 \%$ in the $\phi=8$ flow and $16 \%$ in the $\phi=1 / 8$ flow.

Comparing the schlieren image data in figure 5 and figure 8 reveals that tripping the high-speed-side boundary layer has also changed the large-scale structure, far downstream. For the tripped flow, a decrease in the two-dimensional organization of the flow is evident, with the spanwise optical integration obscuring the local largescale structures, in the upstream half of the flow, which are only evident further downstream. The inferred 'marching' scalar p.d.f. behaviour for this flow is observed along with this decrease in two-dimensional organization, as also noted by Rogers $\&$ Moser (1994) in their numerical simulations. In the context of the large-scaleentrainment model for this flow (Dimotakis 1986), a change in the entrained-fluid 


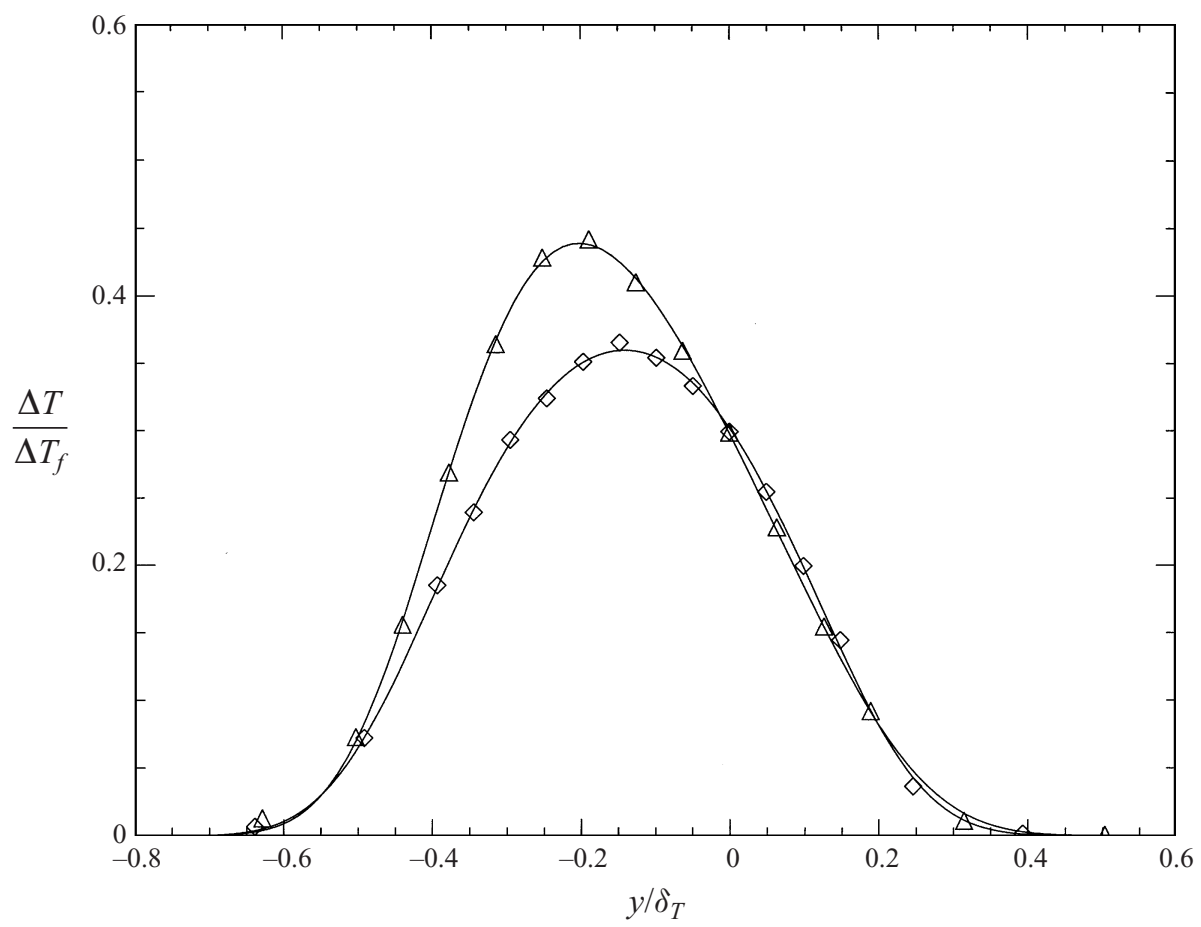

FiguRE 10. Normalized temperature-rise data for tripped and untripped boundary layers at $\phi=1 / 8$. Transverse coordinate normalized by $\delta_{T}$. Legend as figure 9 .

mole ratio, $E_{n}$, is expected. While this was not measured, one can infer the mixed-fluid molar composition ratio,

$$
\mathscr{E}_{n}=\frac{\left(\delta_{p} / \delta_{T}\right)_{\phi_{0}}}{\left(\delta_{p} / \delta_{T}\right)_{1 / \phi_{0}}} .
$$

In the limit of large $\phi_{0}$, this measures the moles of high-speed freestream fluid, per mole of low-speed freestream fluid, that are molecularly mixed in the layer. The two ratios, $E_{n}$ and $\mathscr{E}_{n}$, need not have the same value. The former measures the entrainedfluid ratio, at the largest scales of the flow, whereas the latter measures the mixed-fluid ratio. Using the tripped and untripped shear layer temperature-rise measurements, we find from (18),

$$
\left(\mathscr{E}_{n}\right)_{\text {untripped }} \simeq 1.52, \quad \text { vs. } \quad\left(\mathscr{E}_{n}\right)_{\text {tripped }} \simeq 1.39
$$

i.e. a shift to a lower mixed-fluid composition ratio (closer to symmetric).

The increase in the large-scale-structure spacing-to-position ratio induced by the trip, evident in the schlieren data, suggests that the change in $E_{n}$ is towards larger values, i.e. in the opposite direction. In addition, the visual-thickness growth rate, at least as derived from a few, single-realization, temporally-resolved schlieren images, appears to be slightly larger for the tripped flow (figure 8) than that for the untripped flow (figure 5), i.e. opposite to the reduction noted for the temporally-averaged $1 \%$ temperature-rise thickness, $\delta_{T}$. The $1 \%$ temperature-rise thickness, $\delta_{T}$, has been reported to be close to the visual thickness, $\delta_{\text {vis }}$. To a first approximation, both rely on the bounding extent of mixed fluid. As can be appreciated, however, the two measures differ in the way the distribution of unmixed fluid affects them. The 


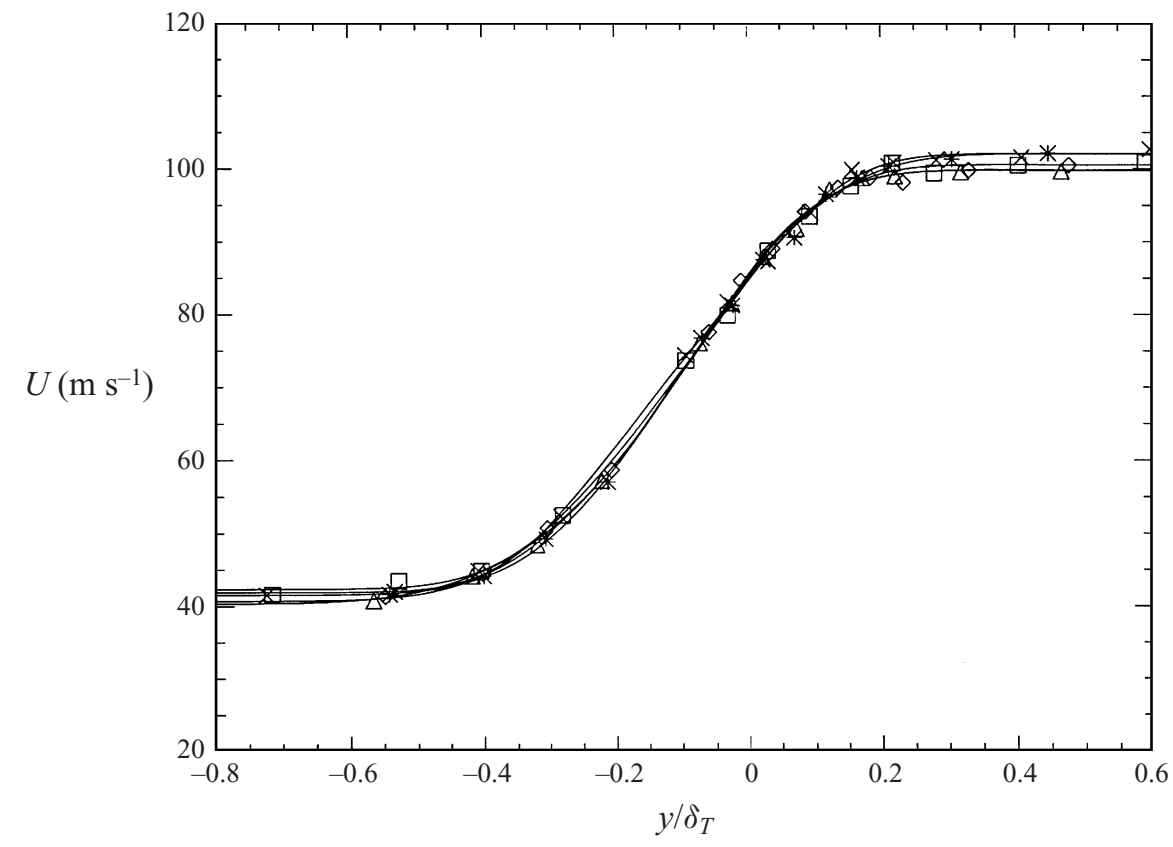

FIGURE 11. Velocity profiles for both untripped and tripped flows. Transverse coordinate normalized by $1 \%$ temperature-rise thickness.

visual thickness, $\delta_{v i s}$, is effectively determined by the transverse extent of the mixedfluid-containing large-scale structures, whereas the $1 \%$ temperature-rise thickness, $\delta_{T}$, depends on the mixed-/unmixed-fluid spatial duty cycle (cf. Mungal \& Dimotakis 1984) near the shear-layer edges, which is sensitive to the large-scale structure itself. Similar considerations apply to the difference between $E_{n}$, the entrained-fluid ratio, and $\mathscr{E}_{n}$, the mixed-fluid composition ratio, which are found to respond in the opposite direction to the tripping. It is interesting that the change in the mixed-fluid distribution across the shear-layer transverse extent is such as to result in a sign difference in the growth-rate change, as registered by the two different measures.

The velocity profiles for the untripped and tripped flows examined thus far are plotted in figure 11 , vs. the normalized transverse coordinate, $y / \delta_{T}$, for comparison. This scaling can be seen to produce a substantial collapse of the mean, streamwise velocity profile at the measuring station, despite the differences between tripped and untripped flows discussed above. This collapse also demonstrates that the transverse length scale, as estimated on the basis of mixed-fluid/temperature-rise data, also scales the velocity field, i.e. that $\delta_{u}(x) \simeq \delta_{T}(x)$, where $\delta_{u}(x)$ would be some appropriate velocity-profile-based transverse width. Finally, the collapse in the scaled profiles in figure 11, indicates that the mean velocity is largely insensitive to the processes responsible for the changes in entrainment and mixing. Conversely, it would be surprising if shear-layer-entrainment and -mixing models based on mean-velocity profiles, e.g. of eddy-viscosity-type, could accommodate, let alone predict, the observed mixing dependence on inflow conditions.

Additional experiments were performed to explore the effects that other near-field perturbations might have, at these nominal conditions. Guided by the results of Lang (1985), in which a large low-speed-side momentum defect (wake) was found to significantly increase the shear-layer growth rate at a freestream speed ratio of $r \simeq 0.5$, 


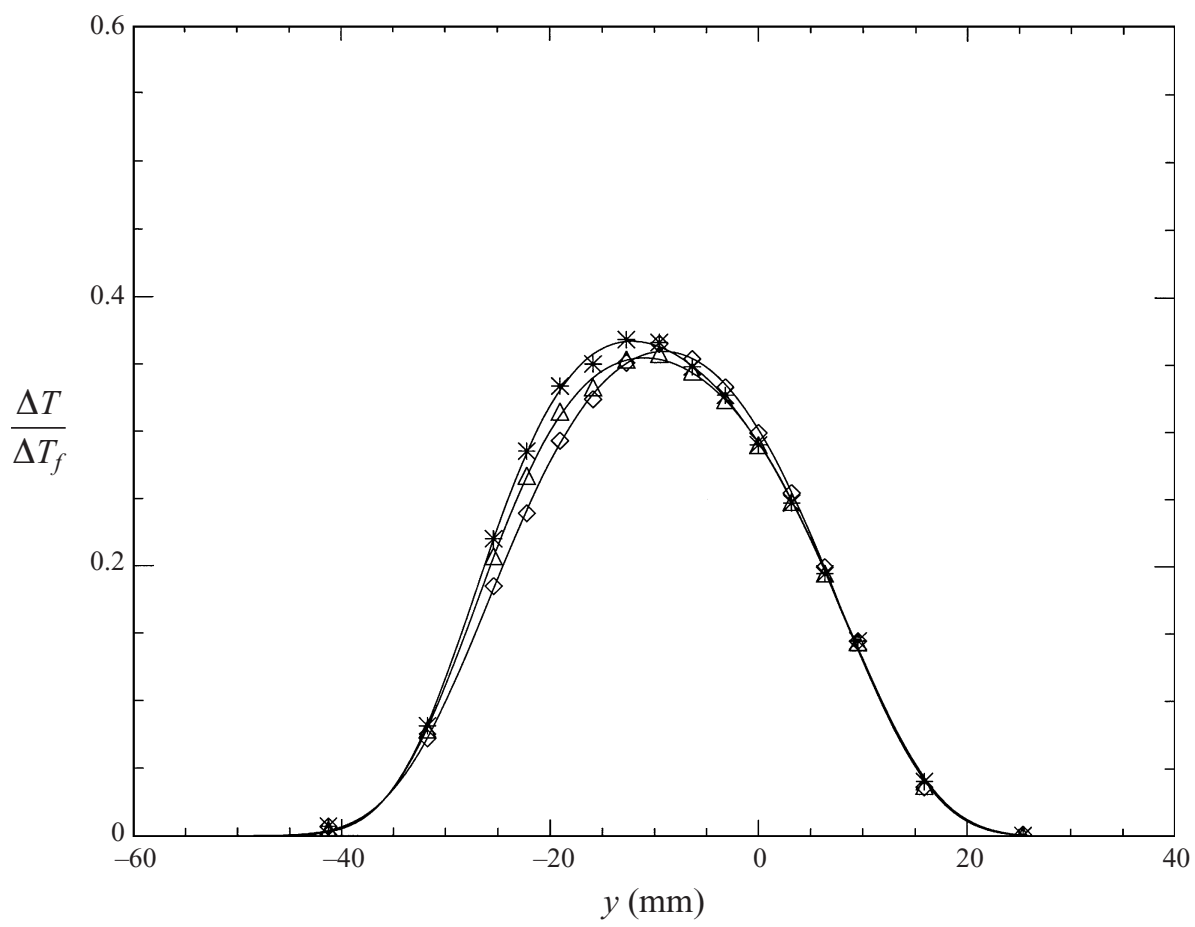

FIGURE 12. Normalized temperature-rise data with various low-speed boundary layer trips. All flows with Case 2 reactant concentrations. Diamonds: Untripped flow. Triangles: $0.8 \mathrm{~mm}$ trip wire. Asterisks: $1.6 \mathrm{~mm}$ trip wire.

two different trip wires were employed on the low-speed side of the splitter plate, with diameters of $0.8 \mathrm{~mm}$ and $1.6 \mathrm{~mm}$, also at $x=-50 \mathrm{~mm}$. The natural low-speed side boundary layer is estimated to be laminar and to have a momentum thickness of $0.15 \mathrm{~mm}$ at this trip location. As seen in figure 12, these low-speed-side perturbations have little, if any, influence on the far-field growth of the layer, in contrast to the significant changes induced by tripping the high-speed splitter-plate boundary layer, and only a weak effect on mixing.

The low-speed-side splitter plate has concave curvature over a portion of its length (cf. figures 1 and 2), and may be susceptible to Taylor-Görtler vortex formation. At the conditions of these experiments, however, the Taylor number is estimated to be approximately 4, significantly less than the stability limit of 7 proposed by Liepmann (1945; cf. also discussion in Schlichting 1968). This indicates that the untripped low-speed boundary layer did not support Taylor-Görtler vortices and was probably laminar. We then infer that the lack of low-speed-side boundary-layer trip influence is not because the naturally-occurring (untripped) low-speed boundary layer is already turbulent. A direct measurement that would reveal the boundary-layer state would not be a simple undertaking in this flow facility and was not attempted.

As a further exploration, both splitter-plate boundary layers were tripped to see if additional effects could be detected (cf. figure 13). In this case, the temperature-rise profile is nearly indistinguishable from that of the flow in which only the high-speed boundary layer was tripped.

Table 3 summarizes the various computed quantities for each of the natural, or tripped, inflow boundary layer configurations. 


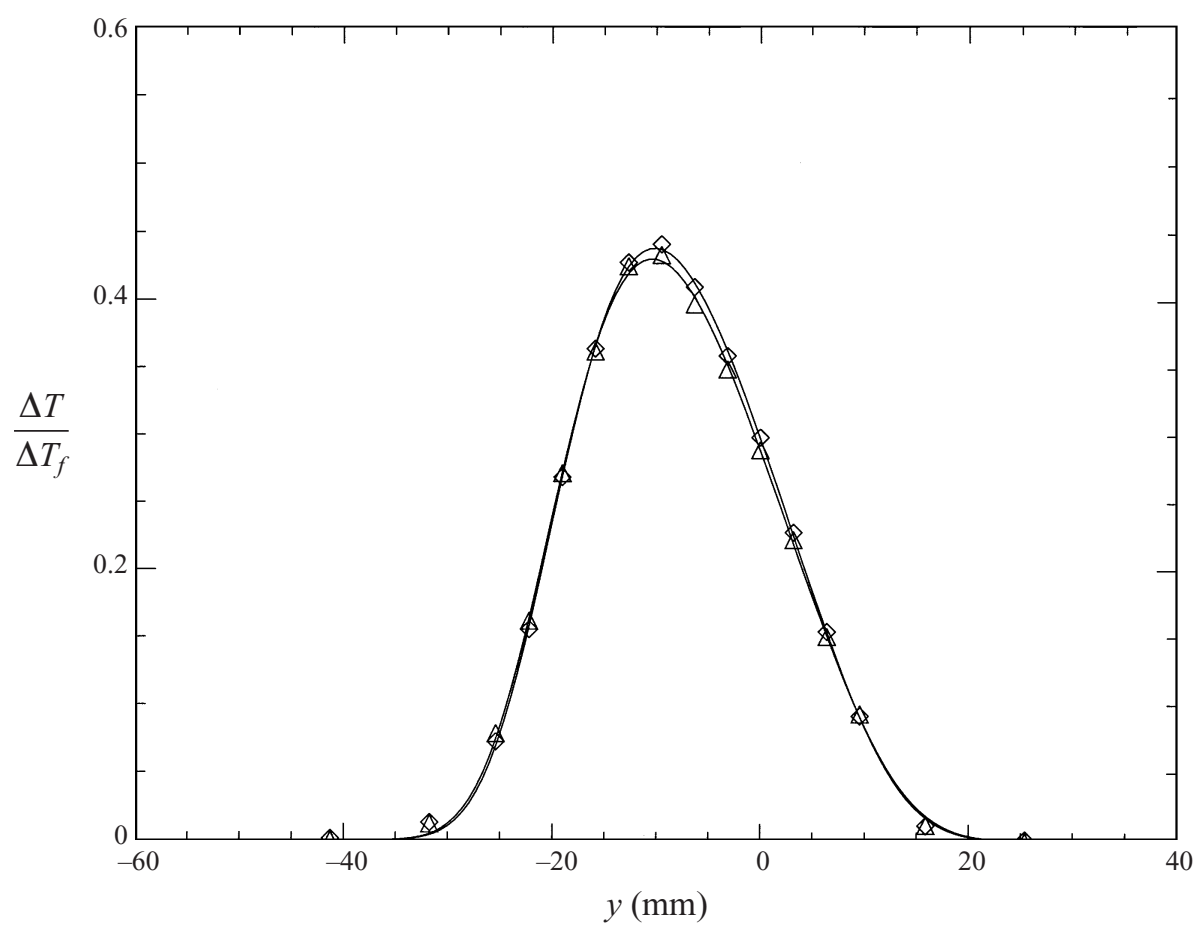

FIGURE 13. Normalized temperature-rise data with various boundary layer trips. Both flows employ Case 2 reactant concentrations, and $0.8 \mathrm{~mm}$-diameter trip wires. Diamonds: Both sides tripped, triangles: High-speed side tripped.

Mixture, perturbation

Case 1, untripped:

Case 1, 0.8 mm HSS trip:

Case 2, untripped:

Case 2, $0.8 \mathrm{~mm}$ HSS trip:

Case 2, $0.8 \mathrm{~mm}$ LSS trip:

Case 2, $1.6 \mathrm{~mm}$ LSS trip:

Case 2, $0.8 \mathrm{~mm}$ trips (both):

Case 3, untripped:

$\begin{array}{cccc}\delta_{T} /(\mathrm{mm}) & \delta_{p} /(\mathrm{mm}) & \delta_{p} / \delta_{T} & R e_{\delta} \times 10^{5} \\ 65.0 & 18.0 & 0.277 & 2.3 \\ 51.1 & 15.0 & 0.293 & 1.8 \\ 64.6 & 11.9 & 0.184 & 2.3 \\ 50.5 & 10.7 & 0.211 & 1.8 \\ 63.7 & 12.0 & 0.189 & 2.2 \\ 63.7 & 12.4 & 0.195 & 2.2 \\ 51.3 & 10.6 & 0.206 & 1.8 \\ 67.4 & 12.3 & 0.181 & 2.4\end{array}$

TABLE 3. Chemically-reacting flow data.

\section{Conclusions}

In the shear-layer flows investigated, the measuring station is well downstream of the minimum recommended distance, as scaled by the initial boundary-layer(s) momentum thickness, the values of the 'pairing parameter' (equation $(5 a)$ ) are well in excess of previously-proposed minimum values, and local Reynolds numbers are well beyond the 'mixing transition', i.e. $R e_{\delta} \gg 10^{4}$, as indicated for asymptotic, self-similar behaviour, mixing, and chemical-product formation.

In assessing the various criteria for the attainment of self-similarity, it could be argued that the Bradshaw, minimum- $x / \theta$ criterion, which was devised for a half-jet, should be modified in the case of a two-stream mixing layer. In the latter case, two 
separating boundary layers are contributed into the near-field region downstream of the splitter-plate trailing edge, with two momentum thicknesses, $\theta_{1}$ and $\theta_{2}$. Perhaps, then, the minimum distance to attain a quasi-self-similar state should be scaled by the sum of the two, i.e. $x /\left(\theta_{1}+\theta_{2}\right)$, which must then be greater than some minimum, possibly the same (equation (3)), value. Other experimental evidence, however, as discussed in the context of the Dimotakis \& Brown (1976) criterion, devised for a two-stream mixing layer, supports the original Bradshaw proposal, i.e. a downstream distance scaled as $x / \theta_{1}$. Additionally, the initial Kelvin-Helmholtz roll-up length, $\ell_{0}$, which launches the subsequent large-scale sequence, is scaled by $\theta_{1}$, as indicated by linear-stability analysis. Finally, in the experimental results presented here, tripping the low-speed boundary layer, which increases its momentum thickness, was found to have no effect on the flow.

At least on the basis of the flow-visualization data presented here, one may conclude that quasi-self-similarity is attained relatively early on, with no evidence of a transition in behaviour at some indicated scaled downstream location, by any of the criteria discussed in the Introduction. This may not be as clear in the tripped-flow case images; an initially-turbulent high-speed boundary layer will not permit the high spanwise coherence to develop from the outset, with the large-scale-structure organization only evident when the span-to-height aspect ratio of the large-scale structures has become small enough, as discussed above. Nevertheless, even in that case, a quasi-linear growth can be seen nearly along the full downstream extent of the developing shear layer.

We note that a free shear layer is a particularly good candidate flow to study the effects of inflow/outflow conditions. Following the initial post-trailing-edge region, the flow is locally only convectively unstable (Huerre \& Monkewitz 1990), with a small absolute-instability region confined to the wake region downstream of the splitter-plate trailing edge (cf. Koch 1985). In the context of convective/absolute instability behaviour, altering the characteristics of the absolute-instability region, that feeds the convectively-unstable flow downstream, may be expected to produce large effects. Secondly, the array of large-scale vortical structures in this flow are all of like sign in their circulation, leading to the strong upstream/downstream coupling noted by Dimotakis \& Brown (1976). Other flows, such as wakes or boundary layers, for example, either have large-scale structures with a circulation of alternating sign, or an antisymmetric vortical image system that presents similarly-attenuated long-range induced-velocity effects.

The shear-layer growth rate as well as molecular mixing were found to depend on inflow conditions; phenomena associated with large- and small-scale behaviour, respectively. The present results corroborate previously-documented findings as regards the effects of inflow (boundary-layer) conditions on shear-layer growth. They also demonstrate, however, that inflow conditions influence small-scale (molecular) mixing in shear layers, far downstream and at high values of the local Reynolds number. Interestingly, scaling the velocity profiles downstream by the shear-layer thickness removes the effect of inflow conditions on the flow, concealing the conspicuous effects on large-scale growth and entrainment, and (molecular) mixing. While it could be argued that small-scale mixing behaviour is slaved to the environment created by the altered large-scales in the flow, the observations documented above indicate that small-scale behaviour is not universal, i.e. is not dictated by local flow variables and Reynolds number.

In conclusion, shear-layer flow data presented here, as well as in previous investigations, indicate that while a quasi-self-similar state can be achieved at high Reynolds 
numbers, within a relatively short downstream extent, this state is a function of inflow conditions. This is at variance with previously-applied criteria for self-similarity, which imply the existence of some universal flow state beyond a minimum scaled downstream distance. In particular, the flow at high Reynolds numbers and away from inflow boundaries is not found to be a unique function of local-flow parameters, such as freestream-velocity and -density ratios, local Reynolds number, etc. as has been argued by George (1989). No support for the existence of a universal, localparameter-defined flow can be gleaned from these investigations. Indeed, the apparent discrepancies between the new results and previous findings, as well as inconsistencies between numerous previous reports, are probably attributable to past interpretations that have implicitly assumed that an inflow-independent, quasi-asymptotic state exists, as discussed in the Introduction.

We wish to acknowledge the contributions and expert assistance of Earl Dahl in support of the Supersonic Shear Layer facility and the execution of these experiments, and of Dan Lang with the data acquisition, computing, and networking environment that made these experiments possible. We would also like to acknowledge two of the referees who commented on the difference in behaviour between the visual and temperature-rise thickness that led to the corresponding discussion in the final text. The work was funded by the Air-Breathing Propulsion program of the Air Force Office of Scientific Research, Grant Nos. F49620-93-1-0338 and F49620-94-1-0353.

\section{REFERENCES}

BAtchelor, G. K. 1953 The Theory of Homogeneous Turbulence. Cambridge University Press.

BATT, R. G. 1975 Some measurements on the effect of tripping the two-dimensional shear layer. AIAA J. 13, 245-247.

Bell, J. H. \& Mehta, R. D. 1990 Development of a two-stream mixing layer from tripped and untripped boundary layers. AIAA J. 28, 2034-2042.

Bernal, L. P., Breidenthal, R. E., Brown, G. L., Konrad, J. H. \& Roshko, A. 1979 On the development of three-dimensional small scales in turbulent mixing layers. 2nd Intl Symp. on Turb. Shear Flows, pp. 305-313. Springer.

Bond, C. L. \& Dimotakis, P. E. 1996 Molecular mixing in high Reynolds number, subsonic, free shear layers. Fall Technical Meeting, Western States Section (Combustion Institute), 28-29 October 1996 (University of Southern California), Paper 96F-099.

BRadshaw, P. 1966 The effect of initial conditions on the development of a free shear layer. J. Fluid Mech. 26, 225-236.

BReIdenthal, R. E. 1978 A chemically reacting turbulent shear layer. PhD thesis, California Institute of Technology.

BREIDENTHAL, R. E. 1981 Structure in turbulent mixing layers and wakes using a chemical reaction. J. Fluid Mech. 109, 1-24.

Broadwell, J. E. \& Mungal, M. G. 1991 Large-scale structures and molecular mixing Phys. Fluids A 3, 1193-1206.

Browand, F. K. \& Latigo, B. O. 1979 Growth of the two-dimensional mixing layer from a turbulent and non-turbulent boundary layer. Phys. Fluids 22, 1011-1019.

Coles, D. 1965 Transition in circular Couette flow. J. Fluid Mech. 21, 385-425.

Dimotakis, P. E. 1986 Two-dimensional shear-layer entrainment. AIAA J. 24, 1791-1796.

Dimotakis, P. E. 1991 Turbulent free shear layer mixing and combustion. In High Speed Flight Propulsion Systems. Progress in Astronautics and Aeronautics, vol. 137, Ch. 5, pp. 265-340.

Dimotakis, P. E. 1993 Some issues on turbulent mixing and turbulence GALCIT Rep. FM93-1a.

Dimotakis, P. E. \& Brown, G. L. 1976 The mixing layer at high Reynolds number: Large-structure dynamics and entrainment. J. Fluid Mech. 78, 535-560 + 2 plates. 
Dimotakis, P. E. \& Hall, J. L. 1987 A simple model for finite chemical kinetics analysis of supersonic turbulent shear layer combustion. AIAA/SAE/ASME/ASEE 23rd Joint Propulsion Meeting, Paper 87-1879.

Dziomba, B. \& Fiedler, H. E. 1985 Effect of initial conditions on two-dimensional free shear layers. J. Fluid Mech. 152, 419-442.

Frieler, C. E. \& Dimotakis, P. E. 1988 Mixing and reaction at low heat release in the nonhomogeneous shear layer. First National Fluid Dynamics Congress, Paper 88-3626.

GeORGE, W. K. 1989 The self-preservation of turbulent flows and its relation to initial conditions and coherent structures. In Advances in Turbulence (ed. W. K. George \& R. Arndt), pp. 39-73. Hemisphere.

HaLl, J. L. 1991 An experimental investigation of structure, mixing and combustion in compressible turbulent shear layers. PhD thesis, California Institute of Technology.

Hall, J. L. \& Dimotakis, P. E. 1989 Design overview of the supersonic hydrogen-fluorine facility (V4.0) GALCIT Internal Rep. 30 August 1989.

Hermanson, J. C. \& Dimotakis, P. E. 1989 Effects of heat release in a turbulent reacting shear layer. J. Fluid Mech. 199, 333-375.

Ho, C.-M. \& HuAng, L.-S. 1982 Subharmonics and vortex merging in mixing layers. J. Fluid Mech. 119, 443-473.

Ho, C.-M. \& Huerre, P. 1984 Perturbed free shear layers. Ann. Rev. Fluid Mech. 16, 365-424.

HuANG, L.-S. \& Ho, C.-M. 1990 Small-scale transition in a plane mixing layer. J. Fluid Mech. 210, $475-500$.

Huerre, P. \& Monkewitz, P. A. 1985 Absolute and convective instabilities in free shear layers. $J$. Fluid Mech. 159, 151-168.

Hussain, A. K. M. F. 1977 Initial condition effect on free turbulent shear flows. In Proc. Structure and Mechanisms of Turbulence I, pp. 103-107. Springer.

ISLAND, T. C. 1997 Quantitative scalar measurements and mixing enhancement in compressible shear layers. Stanford HTGL Rep. TSD-104.

Karasso, P. S. \& Mungal, M. G. 1996 Scalar mixing and reaction in plane liquid shear layers. J. Fluid Mech. 323, 23-63.

КосH, W. 1985 Local instability characteristics and frequency determination of self-exicited wake flows. J. Sound Vib. 99, 53-83.

KonRaD, J. H. 1976 An experimental investigation of mixing in two-dimensional turbulent shear flows with applications to diffusion-limited chemical reactions. PhD thesis, California Institute of Technology.

Koochesfahani, M. M. \& Dimotakis, P. E. 1986 Mixing and chemical reactions in a turbulent liquid mixing layer. J. Fluid Mech. 170, 83-112.

Koochesfahani, M. M., Dimotakis, P. E. \& Broadwell, J. E. 1985 A 'flip' experiment in a chemically reacting turbulent mixing layer. AIAA J. 23, 1191-1194.

Koochesfahani, M. \& MacKinnon, C. 1991 Influence of forcing on the compositions of mixed fluid in a two-stream shear layer. Phys. Fluids A 3, 1135-1142.

LANG, D. B. 1985 Laser Doppler velocity and vorticity measurements in turbulent shear layers. PhD thesis, California Institute of Technology.

LiEPMANN, H. W. 1945 Investigation of boundary layer transition on concave walls. NACA Wartime Rep. W-87.

LiepmanN, H. W. \& Laufer, J. 1947 Investigations of free turbulent mixing. NACA TN-1257.

Miller, P. L. 1991 Mixing in high Schmidt number turbulent jets. PhD thesis, California Institue of Technology.

Mungal, M. G. \& Dimotakis, P. E. 1984 Mixing and combustion with low heat release in a turbulent mixing layer. J. Fluid Mech. 148, 349-382.

Mungal, M. G., Hermanson, J. C. \& Dimotakis, P. E. 1985 Reynolds number effects on mixing and combustion in a reacting shear layer AIAA J. 23, 1418-1423.

NARAYANAN, S. \& Hussain, F. 1996 Measurements of spatiotemporal dynamics in a forced plane mixing layer. J. Fluid Mech. 320, 71-115.

Oster, D. \& Wygnanski, I. 1982 The forced mixing layer between parallel streams. J. Fluid Mech. 123, 91-130.

Oster, D., WygnANSKI, I. \& Fiedler, H. 1976 Some preliminary observations on the effect of initial 
conditions on the structure of the two-dimensional turbulent mixing layer. In Turbulence in Internal Flows, Project SQUID Workshop (ed. S. N. B. Murthy), pp. 67-87. Hemisphere.

RoBerts, F. A. 1985 Effects of a periodic disturbance on structure and mixing in turbulent shear layers and wakes. $\mathrm{PhD}$ thesis, California Institute of Technology.

Roberts, F. A. \& Roshko, A. 1985 Effects of periodic forcing on mixing in turbulent shear layers and wakes. AIAA Shear Flow Control Conference, Paper 85-0570.

Rogers, M. M. \& Moser, R. D. 1992 The three-dimensional evolution of a plane mixing layer: the Kelvin-Helmholtz rollup. J. Fluid Mech. 243, 183-226.

Rogers, M. M. \& Moser, R. D. 1994 Direct simulation of a self-similar turbulent mixing layer. Phys. Fluids 6, 903-923.

Schlichting, H. 1968 Boundary-Layer Theory, 6th edn. McGraw-Hill.

SETtLES, G. S. 1985 Colour-coding schlieren techniques for the optical study of heat and fluid flow. Intl J. Heat Fluid Flow 6, 3-15.

Weisbrot, I., EInAV, S. \& Wygnanski, I. 1982 The non unique rate of spread of the two-dimensional mixing layer. Phys. Fluids 25, 1691-1693.

White, F. M. 1974 Viscous Fluid Flow. McGraw-Hill.

Wilke, C. R. 1950 A viscosity equation for gas mixtures. J. Chem. Phys. $18,517$.

WygnANSKi, I., ChAmpagne, F. \& MARASLI, B. 1986 On the large-scale structures in two-dimensional, small-deficit turbulent wakes. J. Fluid Mech. 38, 577-612.

Wygnanski, I. \& Fiedler, H. E. 1970 The two-dimensional mixing region. J. Fluid Mech. 41, $327-361$

WygnANSKi, I., Oster, D. \& Fiedler, H. 1979 A forced, plane, turbulent mixing-layer: a challenge for the predictor. 2nd Intl Symp. on Turb. Shear Flows, pp. 314-326. Springer.

YaO, L. S. \& Ghosh Moulic, S. 1995 Nonlinear instability of traveling waves with a continuous spectrum. Intl J. Heat Mass Transfer 38, 1751-1772. 